\title{
Application of Conformable Sumudu Decomposition Method for Solving Conformable Fractional Coupled Burger's Equation
}

\author{
Hassan Eltayeb (iD) and Said Mesloub \\ Mathematics Department, College of Science, King Saud University, P.O. Box 2455, Riyadh 11451, Saudi Arabia
}

Correspondence should be addressed to Hassan Eltayeb; hgadain@ksu.edu.sa

Received 13 October 2020; Revised 4 November 2020; Accepted 13 February 2021; Published 8 March 2021

Academic Editor: Richard I. Avery

Copyright (c) 2021 Hassan Eltayeb and Said Mesloub. This is an open access article distributed under the Creative Commons Attribution License, which permits unrestricted use, distribution, and reproduction in any medium, provided the original work is properly cited.

\begin{abstract}
The conformable double Sumudu decomposition method (CDSDM) is a combination of decomposition method (DM) and a conformable double Sumudu transform. It is an approximate analytical method, which can be used to solve linear and nonlinear partial differential equations. In this work, one-dimensional conformable functional Burger's equation has been solved by applying conformable double Sumudu decomposition. Two examples are used to illustrate the method.
\end{abstract}

\section{Introduction}

Burgers' equation is represented by nonlinear wave motion with linear diffusion. These equations have appeared in the area of applied sciences such as fluid mechanics and mathematical modeling. The importance of getting the exact or approximate solutions of Burgers' equations in mathematics and physics is still a significant problem that needs new methods to discover exact or approximate solutions. Theoretical solution of Burger equations, based on Fourier series analysis, using the appropriate initial and boundary conditions was discussed in [1]. The authors in [2] studied different exact solutions of Burger's like equations and their classifications. The author in [3] discussed $(2+1)$-dimensional time-fractional Bogoyavlensky-Konopelchenko (BK) equation by using a subequation method that is predicated on the Riccati equation. The new wave solutions of the fractional Camassa-Holm equation are studied by applying a new extended direct algebraic method (see [4]). The wave solutions of the time-fractional Benjamin-Ono equation are established by using the Jacobi elliptic function expansion method for more details (see [5]). The solitary and traveling wave solutions for the time-fractional $\mathrm{pKP}$ equation have been obtained by applying the new direct algebraic method by means of the conformable derivative see [6]. Many powerful methods were used to solve nonlinear partial differential equations, such as the fractional Sumudu transform $[7,8]$. The exact solutions for time-fractional Burgers' equations were studied by the first integral method [9]. The authors in [10] employed the generalized two-dimensional differential transform method (DTM) and obtained the solution for the coupled Burgers' equations with space- and timefractional derivatives. In recent time, the new definition of the conformable derivative has been introduced for more specifics; we refer to [11-13]. Furthermore, the authors in $[11,14]$ are solved the fractional differential equations by conformable Laplace transform technique. In [15], the researchers applied the conformable double Laplace transform method and obtained the solution for the fractional partial differential equations. The conformable double Laplace decomposition method [16] has been proposed to obtain exact and approximate solutions of regular and singular one-dimensional conformable fractional coupled burgers' equations. The exact solutions of the time-fractional Burgers' equations were established by using the first integral method (see [9]). We introduce a new method called the conformable double Sumudu decomposition method (CDSDM) for solving the nonlinear equations in the present paper. The proposed method is an elegant combination of the conformable double Sumudu transform method and the decomposition method. This paper considers the effectiveness of the conformable double Sumudu decomposition 
method (CDSDM) in solving regular and singular onedimensional conformable fractional coupled burgers' equations, and the advantage of this method is easy to apply for solving fractional nonlinear partial differential equations. In the following, we list some definitions from the conformable derivatives which are used further in this paper.

Definition 1 (see $[13,17-19]$ ). Given a function $f:(0, \infty)$ $\longrightarrow R$, then the conformable fractional derivative of $f$ of order $\alpha$ is defined by

$$
\frac{d^{\alpha}}{d t^{\alpha}} f(t)=\lim _{\varepsilon \longrightarrow 0} \frac{f\left(t+\varepsilon t^{1-\alpha}\right)-f(t)}{\varepsilon}, \quad t>0,0<\alpha \leq 1 .
$$

Definition 2 (see [20]). Given a function $f\left(x^{\alpha} / \alpha, t^{\beta} / \beta\right): R \times($ $0, \infty) \longrightarrow R$. Then, the conformable space fractional partial derivative of order $\alpha$ a function $f\left(x^{\alpha} / \alpha, t^{\beta} / \beta\right)$ is defined as follows:

$$
\frac{\partial^{\alpha}}{\partial x^{\alpha}} f\left(\frac{x^{\alpha}}{\alpha}, \frac{t^{\beta}}{\beta}\right)=\lim _{\varepsilon \rightarrow 0} \frac{f\left(x^{\alpha} / \alpha,+\varepsilon x^{1-\alpha}, t\right)-f\left(x^{\alpha} / \alpha, t^{\beta} / \beta\right)}{\varepsilon}, \quad \frac{x^{\alpha}}{\alpha}, \frac{t^{\beta}}{\beta}>0,0<\alpha, \beta \leq 1,
$$

Definition 3 (see [20]). Given a function $f\left(x^{\alpha} / \alpha, t^{\beta} / \beta\right): R \times($ $0, \infty) \longrightarrow R$. Then, the conformable time fractional partial derivative of order $\beta$ a function $f\left(x^{\alpha} / \alpha, t^{\beta} / \beta\right)$ is defined as follows:

$\frac{\partial^{\beta}}{\partial t^{\beta}} f\left(\frac{x^{\alpha}}{\alpha}, \frac{t^{\beta}}{\beta}\right)=\lim _{\sigma \rightarrow 0} \frac{f\left(x^{\alpha} / \alpha, t^{\beta} / \beta+\sigma t^{1-\beta}\right)-f\left(x^{\alpha} / \alpha, t^{\beta} / \beta\right)}{\sigma}, \quad \frac{x^{\alpha}}{\alpha}, \frac{t^{\beta}}{\beta}>0,0<\alpha, \beta \leq 1$,

The following are the conformable fractional derivative of certain functions:

Example 4 (see [16]).

$$
\begin{aligned}
& \frac{\partial^{\alpha}}{\partial x^{\alpha}}\left(\frac{x^{\alpha}}{\alpha}\right)\left(\frac{t^{\beta}}{\beta}\right)=\left(\frac{t^{\beta}}{\beta}\right), \quad \frac{\partial^{\alpha}}{\partial x^{\alpha}}\left(\frac{x^{\alpha}}{\alpha}\right)^{n}\left(\frac{t^{\beta}}{\beta}\right)=n\left(\frac{x^{\alpha}}{\alpha}\right)^{n-1}\left(\frac{t^{\beta}}{\beta}\right), \\
& \frac{\partial^{\beta}}{\partial t^{\beta}}\left(\frac{x^{\alpha}}{\alpha}\right)\left(\frac{t^{\beta}}{\beta}\right)=\left(\frac{x^{\alpha}}{\alpha}\right), \quad \frac{\partial^{\beta}}{\partial t^{\beta}}\left(\frac{x^{\alpha}}{\alpha}\right)^{n}\left(\frac{t^{\beta}}{\beta}\right)^{m}=m\left(\frac{x^{\alpha}}{\alpha}\right)^{n}\left(\frac{t^{\beta}}{\beta}\right)^{m-1}, \\
& \frac{\partial^{\beta}}{\partial t^{\beta}}\left(\sin \left(\frac{x^{\alpha}}{\alpha}\right) \sin \left(\frac{t^{\beta}}{\beta}\right)\right)=\sin \left(\frac{x^{\alpha}}{\alpha}\right) \cos \left(\frac{t^{\beta}}{\beta}\right), \\
& \frac{\partial^{\alpha}}{\partial x^{\alpha}}\left(\sin a\left(\frac{x^{\alpha}}{\alpha}\right) \sin \left(\frac{t^{\beta}}{\beta}\right)\right)=a \cos \left(\frac{x^{\alpha}}{\alpha}\right) \sin \left(\frac{t^{\beta}}{\beta}\right), \\
& \frac{\partial^{\alpha}}{\partial x^{\alpha}}\left(e^{\lambda\left(x^{\alpha} / \alpha\right)+\left(\tau t^{\beta} / \beta\right)}\right)=\lambda e^{\lambda\left(x^{\alpha} / \alpha\right)+\left(\tau t^{\beta} / \beta\right),} \\
& \frac{\partial^{\beta}}{\partial t^{\beta}}\left(e^{\lambda\left(x^{\alpha} / \alpha\right)+\left(\tau t^{\beta} / \beta\right)}\right)=\tau e^{\lambda\left(x^{\alpha} / \alpha\right)+\left(\tau t^{\beta} / \beta\right)} .
\end{aligned}
$$

We introduce the conformable double Sumudu transform and solve the conformable fractional coupled burgers' equation by using this new definition.
Definition 5. Over the set of function

$$
A=\left\{f\left(\frac{t^{\beta}}{\beta}\right): \exists M, \tau_{1}, \tau_{2}>0,\left|f\left(\frac{t^{\beta}}{\beta}\right)\right|<M e \frac{\left|t^{\beta} / \beta\right|}{\tau_{j}}, \text { if } t \in(-1)^{j} \times[0, \infty)\right\},
$$

the conformable Sumudu transform is given by

$$
S_{t}^{\beta}\left[f\left(\frac{t^{\beta}}{\beta}\right)\right]=F_{\beta}(u)=\int_{0}^{\infty} f\left(\frac{t^{\beta}}{\beta}\right) e^{-(1 / u)\left(t^{\beta} / \beta\right)} t^{\beta-1} d t, \quad u \in\left(-\tau_{1}, \tau_{2}\right) .
$$

Definition 6. Let $f\left(x^{\alpha} / \alpha, t^{\beta} / \beta\right)$ be function we defined conformable double Sumudu transform of function $f\left(x^{\alpha} / \alpha, t^{\beta}\right)$ $\beta), x^{\alpha} / \alpha, t^{\beta} / \beta \in \mathbb{R}^{+}$is given by

$$
\begin{aligned}
F_{\alpha, \beta}(u, v) & =S_{x}^{\alpha} S_{t}^{\beta}\left[f\left(\frac{x^{\alpha}}{\alpha}, \frac{t^{\beta}}{\beta}\right)\right] \\
& =\frac{1}{u v} \int_{0}^{\infty} \int_{0}^{\infty} e^{-\left((1 / u)\left(x^{\alpha} / \alpha\right)+(1 / v)\left(t^{\beta} \beta\right)\right)} f\left(\frac{x^{\alpha}}{\alpha}, \frac{t^{\beta}}{\beta}\right) x^{\alpha-1} t^{\beta-1} d x d t
\end{aligned}
$$

or

$$
\begin{aligned}
F_{\alpha, \beta}(u, v) & =S_{x}^{\alpha} S_{t}^{\beta}\left[f\left(\frac{x^{\alpha}}{\alpha}, \frac{t^{\beta}}{\beta}\right)\right] \\
& =\int_{0}^{\infty} \int_{0}^{\infty} e^{-\left(x^{\alpha} / \alpha+t^{\beta} / \beta\right)} f\left(u \frac{x^{\alpha}}{\alpha}, v \frac{t^{\beta}}{\beta}\right) x^{\alpha-1} t^{\beta-1} d x d t .
\end{aligned}
$$

where $S_{x}^{\alpha} S_{t}^{\beta}$ represent the conformable double Sumudu transform. The relation between the usual and the conformable double Sumudu transforms is given below.

Example 7. The double conformable Sumudu transform for certain functions are given by

$$
\begin{aligned}
& S_{x}^{\alpha} S_{t}^{\beta}\left[\left(\frac{x^{\alpha}}{\alpha}\right)^{n}\left(\frac{t^{\beta}}{\beta}\right)^{m}\right]=S_{x} S_{t}\left[(x)^{n}(t)^{m}\right]=n ! m ! u^{n} v^{m}, \\
& S_{x}^{\alpha} S_{t}^{\beta}\left[\left(e^{\lambda \frac{x^{\alpha}}{\alpha}+\frac{\tau t^{\beta}}{\beta}}\right]=S_{x} S_{t}\left[\left(e^{\lambda x+\tau t}\right]=\frac{1}{(1-\lambda u)(1-\tau v)},\right.\right. \\
& S_{x}^{\alpha} S_{t}^{\beta}\left[\left(\sin \left(\lambda \frac{x^{\alpha}}{\alpha}\right) \sin \left(\tau \frac{t^{\beta}}{\beta}\right)\right]=S_{x} S_{t}[(\sin (\lambda x) \sin (\lambda t)]\right. \\
& =\frac{\lambda u}{1+\lambda^{2} u^{2}} \frac{\lambda v}{1+\tau^{2} v^{2}}, \\
& S_{x}^{\alpha} S_{t}^{\beta}\left[\cos \left(\frac{x^{\alpha}}{\alpha}\right) \cos \left(\frac{t^{\beta}}{\beta}\right)\right]=S_{x} S_{t}[(\cos (\lambda x) \cos (\lambda t)] \\
& =\frac{1}{1+\lambda^{2} u^{2}} \frac{1}{1+\lambda^{2} v^{2}} .
\end{aligned}
$$


Now, we present the conditions for the existence of the conformable double Sumudu transform:

If $f\left(x^{\alpha} / \alpha, t^{\beta} / \beta\right)$ is an exponential order $a$ and $b$ as $x^{\alpha} / \alpha$ $\longrightarrow \infty, t^{\beta} / \beta \longrightarrow \infty$ and if $\exists K>0$, then for all $x^{\alpha} / \alpha>X$ and $t^{\beta} / \beta>T$,

$$
\left|f\left(\frac{x^{\alpha}}{\alpha}, \frac{t^{\beta}}{\beta}\right)\right| \leq K e^{a\left(x^{\alpha} / \alpha\right)+b\left(t^{\beta} / \beta\right)},
$$

and we write

$$
f\left(\frac{x^{\alpha}}{\alpha}, \frac{t^{\beta}}{\beta}\right)=O\left(e^{a\left(x^{\alpha} / \alpha\right)+b\left(t^{\beta} / \beta\right)}\right) \text { as } \frac{x^{\alpha}}{\alpha} \longrightarrow \infty, \frac{t^{\beta}}{\beta} \longrightarrow \infty
$$

equivalently,

$$
\begin{aligned}
& \underset{\frac{x^{\alpha}}{\alpha} \longrightarrow \infty}{\lim _{\frac{t^{\beta}}{\beta} \longrightarrow \infty}} e^{-(1 / u)\left(x^{\alpha} / \alpha\right)-(1 / \eta)\left(t^{\beta} / \beta\right)}\left|f\left(\frac{x^{\alpha}}{\alpha}, \frac{t^{\beta}}{\beta}\right)\right|=K \\
& \frac{x^{\alpha}}{\lim _{\beta}} e^{-((1 / u)-a)\left(x^{\alpha} / \alpha\right)-((1 / \eta)-b)\left(t^{\beta} / \beta\right)}=0, \\
& \frac{t^{3}}{\beta} \longrightarrow \infty
\end{aligned}
$$

where $(1 / u)>a$ and $(1 / \eta)>b$. The function $f\left(x^{\alpha} / \alpha, t^{\beta} / \beta\right)$ does not grow faster than $K e^{a\left(x^{\alpha} / \alpha\right)+b\left(t^{\beta} / \beta\right)}$ as $\left(x^{\alpha} / \alpha\right) \longrightarrow \infty$, ( $\left.t^{\beta} / \beta\right) \longrightarrow \infty$.

Theorem 8. The function $f\left(x^{\alpha} / \alpha, t^{\beta} / \beta\right)$ is defined on $(0, X)$ and $(0, T)$ and of exponential order $e^{a\left(x^{\alpha} / \alpha\right)+b\left(t^{\beta} / \beta\right)}$; then the conformable Sumudu transform of $f\left(x^{\alpha} / \alpha, t^{\beta} / \beta\right)$ exists for all $\operatorname{Re} 1 / u>1 / \mu, \operatorname{Re} 1 / v>1 / \eta$.

Proof. From equation (29), we obtain

$$
\begin{aligned}
\left|F_{\alpha, \beta}(u, v)\right| & =\left|\frac{1}{u v} \int_{0}^{\infty} \int_{0}^{\infty} e^{-(1 / u)\left(x^{\alpha} / \alpha\right)+(1 / v)\left(t^{\beta} / \beta\right)} f\left(\frac{x^{\alpha}}{\alpha}, \frac{t^{\beta}}{\beta}\right) x^{\alpha-1} t^{\beta-1} d x d t\right| \\
& \leq K\left|\frac{1}{u} \int_{0}^{\infty} e^{-((1 / u)-a)\left(x^{\alpha} / \alpha\right)-((1 / v)-b)\left(t^{\beta} / \beta\right)} x^{\alpha-1} t^{\beta-1} d x d t\right| \\
& =\frac{k}{(1-a u)(1-b v)} .
\end{aligned}
$$

For $\operatorname{Re} 1 / u>1 / \mu, \operatorname{Re} 1 / v>1 / \eta$, from equation (13), we get

$$
\begin{aligned}
\lim _{\frac{x^{\alpha}}{\alpha}} & \longrightarrow \infty \\
\frac{t^{\beta}}{\beta} & \longrightarrow \infty
\end{aligned}\left|F_{\alpha, \beta}(u, v)\right|=
$$

or

$$
\begin{aligned}
\lim _{\frac{x^{\alpha}}{\alpha}} & \longrightarrow \infty \\
\frac{t^{\beta}}{\beta} & \longrightarrow \infty
\end{aligned}
$$

Now the next result deals with the multiplication of the function $f\left(x^{\alpha} / \alpha, t^{\beta} / \beta\right)$ by $x^{\alpha} / \alpha, t^{\beta} / \beta$.

Theorem 9. If conformable double Sumudu transform of the function $f\left(x^{\alpha} / \alpha, t^{\beta} / \beta\right)$ is given by $F_{\alpha, \beta}(u, v)=S_{x}^{\alpha} S_{t}^{\beta}\left[f\left(x^{\alpha} / \alpha\right.\right.$, $\left.\left.t^{\beta} / \beta\right)\right]$, then the conformable double Sumudu transforms of the functions

$$
\frac{x^{\alpha}}{\alpha} f\left(\frac{x^{\alpha}}{\alpha}, \frac{t^{\beta}}{\beta}\right), \frac{t^{\beta}}{\beta} f\left(\frac{x^{\alpha}}{\alpha}, \frac{t^{\beta}}{\beta}\right),\left(\frac{x^{\alpha}}{\alpha}\right)^{2} f\left(\frac{x^{\alpha}}{\alpha}, \frac{t^{\beta}}{\beta}\right),\left(\frac{t^{\beta}}{\beta}\right)^{2} f\left(\frac{x^{\alpha}}{\alpha}, \frac{t^{\beta}}{\beta}\right), \frac{x^{\alpha}}{\alpha} \frac{t^{\beta}}{\beta} f\left(\frac{x^{\alpha}}{\alpha}, \frac{t^{\beta}}{\beta}\right)
$$

are given by

$$
S_{x}^{\alpha} S_{t}^{\beta}\left[\frac{x^{\alpha}}{\alpha} f\left(\frac{x^{\alpha}}{\alpha}, \frac{t^{\beta}}{\beta}\right)\right]=u^{2} \frac{\partial F_{\alpha, \beta}(u, v)}{\partial u}+u F_{\alpha, \beta}(u, v)
$$

$$
S_{x}^{\alpha} S_{t}^{\beta}\left[\frac{t^{\beta}}{\beta} f\left(\frac{x^{\alpha}}{\alpha}, \frac{t^{\beta}}{\beta}\right)\right]=v^{2} \frac{\partial F_{\alpha, \beta}(u, v)}{\partial v}+v F_{\alpha, \beta}(u, v),
$$

$$
\begin{aligned}
S_{x}^{\alpha} S_{t}^{\beta}\left[\left(\frac{x^{\alpha}}{\alpha}\right)^{2} f\left(\frac{x^{\alpha}}{\alpha}, \frac{t^{\beta}}{\beta}\right)\right]= & u^{4} \frac{\partial^{2} F_{\alpha, \beta}(u, v)}{\partial u^{2}}+4 u^{3} \frac{\partial F_{\alpha, \beta}(u, v)}{\partial u} \\
& +2 u^{2} F_{\alpha, \beta}(u, v),
\end{aligned}
$$

$$
\begin{aligned}
S_{x}^{\alpha} S_{t}^{\beta}\left[\left(\frac{t^{\beta}}{\beta}\right)^{2} f\left(\frac{x^{\alpha}}{\alpha}, \frac{t^{\beta}}{\beta}\right)\right]= & v^{4} \frac{\partial^{2} F_{\alpha, \beta}(u, v)}{\partial v^{2}}+4 v^{3} \frac{\partial F_{\alpha, \beta}(u, v)}{\partial v} \\
& +2 v^{2} F_{\alpha, \beta}(u, v),
\end{aligned}
$$

$$
\begin{aligned}
S_{t}^{\beta}\left[\frac{x^{\alpha}}{\alpha} \frac{t^{\beta}}{\beta} f\left(\frac{x^{\alpha}}{\alpha}, \frac{t^{\beta}}{\beta}\right)\right]= & u v \frac{\partial^{2} F_{\alpha, \beta}(u, v)}{\partial u \partial v}+u v^{2} \frac{\partial F_{\alpha, \beta}(u, v)}{\partial v} \\
& +u^{2} v \frac{\partial F_{\alpha, \beta}(u, v)}{\partial u}+u v F_{\alpha, \beta}(u, v),
\end{aligned}
$$

respectively. 
Proof. By using the definition of conformable double Sumudu transform for equation (17), we get

$$
\begin{aligned}
\frac{\partial F_{\alpha, \beta}(u, v)}{\partial u}= & \frac{\partial}{\partial u} \int_{0}^{\infty} \int_{0}^{\infty} \frac{1}{u v} e^{-\left((1 / u)\left(x^{\alpha} / \alpha\right)+(1 / v)\left(t^{\beta} / \beta\right)\right)} f \\
& \cdot\left(\frac{x^{\alpha}}{\alpha}, \frac{t^{\beta}}{\beta}\right) x^{\alpha-1} t^{\beta-1} d x d t, \\
= & \int_{0}^{\infty} \frac{1}{v} e^{-\frac{11^{\beta}}{v \beta}}\left(\int_{0}^{\infty} \frac{\partial}{\partial u} \frac{1}{u} e^{-(1 / u)\left(x^{\alpha} / \alpha\right)} f\left(\frac{x^{\alpha}}{\alpha}, \frac{t^{\beta}}{\beta}\right) x^{\alpha-1} d x\right) t^{\beta-1} d t,
\end{aligned}
$$

by calculating the partial derivative inside brackets, we can easily get

$$
\begin{aligned}
\int_{0}^{\infty} & \frac{\partial}{\partial u} \frac{1}{u} e^{-(1 / u)\left(x^{\alpha} / \alpha\right)} f\left(\frac{x^{\alpha}}{\alpha}, \frac{t^{\beta}}{\beta}\right) x^{\alpha-1} d x \\
= & \int_{0}^{\infty}\left(\frac{1}{u^{3}} \frac{x^{\alpha}}{\alpha}-\frac{1}{u^{2}}\right) e^{-(1 / u)\left(x^{\alpha} / \alpha\right)} f\left(\frac{x^{\alpha}}{\alpha}, \frac{t^{\beta}}{\beta}\right) x^{\alpha-1} d x \\
= & \int_{0}^{\infty} \frac{1}{u^{3}} \frac{x^{\alpha}}{\alpha} e^{-(1 / u)\left(x^{\alpha} / \alpha\right)} f\left(\frac{x^{\alpha}}{\alpha}, \frac{t^{\beta}}{\beta}\right) x^{\alpha-1} d x \\
& -\int_{0}^{\infty} \frac{1}{u^{2}} e^{-(1 / u)\left(x^{\alpha} / \alpha\right)} f\left(\frac{x^{\alpha}}{\alpha}, \frac{t^{\beta}}{\beta}\right) x^{\alpha-1} d x,
\end{aligned}
$$

substituting equation (23) into equation (22), we obtain

$$
\begin{aligned}
\frac{\partial F_{\alpha, \beta}(u, v)}{\partial u}= & \int_{0}^{\infty} \frac{1}{v} e^{-(1 / v)\left(t^{\beta} / \beta\right)}\left(\int_{0}^{\infty} \frac{1}{u^{3}} \frac{x^{\alpha}}{\alpha} e^{-(1 / u)\left(x^{\alpha} / \alpha\right)} f\left(\frac{x^{\alpha}}{\alpha}, \frac{t^{\beta}}{\beta}\right) x^{\alpha-1} d \mathrm{x}\right) t^{\beta-1} d t \\
& -\int_{0}^{\infty} \frac{1}{v} e^{-(1 / v)\left(t^{\beta} / \beta\right)}\left(\int_{0}^{\infty} \frac{1}{u^{2}} e^{-(1 / u)\left(x^{\alpha} / \alpha\right)} f\left(\frac{x^{\alpha}}{\alpha}, \frac{t^{\beta}}{\beta}\right) x^{\alpha-1} d x\right) t^{\beta-1} d t,
\end{aligned}
$$

by applying equation (7), we get equation (17)

$$
S_{x}^{\alpha} S_{t}^{\beta}\left[\frac{x^{\alpha}}{\alpha} f\left(\frac{x^{\alpha}}{\alpha}, \frac{t^{\beta}}{\beta}\right)\right]=u^{2} \frac{\partial F_{\alpha, \beta}(u, v)}{\partial u}+u F_{\alpha, \beta}(u, v)
$$

For equation (19), by using second partial derivative for $F_{\alpha, \beta}(u, v)$ with respect to $u$ and definition of conformable double Sumudu transform, we have

$$
\begin{aligned}
\frac{\partial^{2} F_{\alpha, \beta}(u, v)}{\partial u^{2}}= & \frac{\partial^{2}}{\partial u^{2}} \int_{0}^{\infty} \int_{0}^{\infty} \frac{1}{u v} e^{-\left((1 / u)\left(x^{\alpha} / \alpha\right)+(1 / v)\left(t^{\beta} / \beta\right)\right)} f\left(\frac{x^{\alpha}}{\alpha}, \frac{t^{\beta}}{\beta}\right) x^{\alpha-1} t^{\beta-1} d x d t, \\
= & \infty \\
0 & \frac{1}{v} e^{-(1 / v)\left(t^{\beta} / \beta\right)}\left(\int_{0}^{\infty} \frac{\partial^{2}}{\partial u^{2}}\left(\frac{1}{u} e^{-(1 / u)\left(x^{\alpha} / \alpha\right)}\right) f\left(\frac{x^{\alpha}}{\alpha}, \frac{t^{\beta}}{\beta}\right) x^{\alpha-1} d x\right) t^{\beta-1} d t, \\
= & \int_{0}^{\infty} \frac{1}{v} e^{-(1 / v)\left(t^{\beta} / \beta\right)}\left(\int_{0}^{\infty}\left(\frac{1}{u^{5}}\left(\frac{x^{\alpha}}{\alpha}\right)^{2}-\frac{3}{u^{4}} \frac{x^{\alpha}}{\alpha}\right) e^{-(1 / u)\left(x^{\alpha} / \alpha\right)} f\right. \\
& \left.\cdot\left(\frac{x^{\alpha}}{\alpha}, \frac{t^{\beta}}{\beta}\right) x^{\alpha-1} d x\right) t^{\beta-1} d t-\int_{0}^{\infty} \frac{1}{v} e^{-(1 / v)\left(t^{\beta} / \beta\right)} \\
& \cdot\left(\int_{0}^{\infty}\left(\frac{1}{u^{4}}\left(\frac{x^{\alpha}}{\alpha}\right)-\frac{2}{u^{3}}\right) e^{-(1 / u)\left(x^{\alpha} / \alpha\right)} f\left(\frac{x^{\alpha}}{\alpha}, \frac{t^{\beta}}{\beta}\right) x^{\alpha-1} d x\right) t^{\beta-1} d t .
\end{aligned}
$$

Applying equations (7) and (19), we obtain equation (19)

$$
\begin{aligned}
S_{x}^{\alpha} S_{t}^{\beta}\left[\left(\frac{x^{\alpha}}{\alpha}\right)^{2} f\left(\frac{x^{\alpha}}{\alpha}, \frac{t^{\beta}}{\beta}\right)\right]= & u^{4} \frac{\partial^{2} F_{\alpha, \beta}(u, v)}{\partial u^{2}}+4 u^{3} \frac{\partial F_{\alpha, \beta}(u, v)}{\partial u} \\
& +2 u^{2} F_{\alpha, \beta}(u, v) .
\end{aligned}
$$

Similarly, in the same way, one can prove equations (18), (20), and (21).

The conformable double Sumudu transform of the first partial derivative with respect to $x$ and $t$ is given by

$$
\begin{aligned}
& S_{x}^{\alpha} S_{t}^{\beta}\left[\frac{\partial^{\alpha} \psi}{\partial x^{\alpha}}\right]=\frac{1}{u} \Psi_{\alpha, \beta}(u, v)-\frac{1}{u} \Psi_{\beta}(0, v), \\
& S_{x}^{\alpha} S_{t}^{\beta}\left[\frac{\partial^{\beta} \psi}{\partial t^{\beta}}\right]=\frac{1}{v} \Psi_{\alpha, \beta}(u, v)-\frac{1}{v} \Psi_{\alpha}(u, 0),
\end{aligned}
$$

and the conformable double Sumudu transform of second partial derivative concerning $x$ and $t$ is denoted by

$$
\begin{aligned}
& S_{x}^{\alpha} S_{t}^{\beta}\left[\frac{\partial^{2 \alpha} \psi}{\partial x^{2 \alpha}}\right]=\frac{1}{u^{2}} \Psi_{\alpha, \beta}(u, v)-\frac{1}{u^{2}} \Psi_{\beta}(0, v)-\frac{1}{u} S_{t}^{\beta}\left(\frac{\partial^{\alpha} \psi\left(0, t^{\beta} / \beta\right)}{\partial x^{\alpha}}\right) \\
& S_{x}^{\alpha} S_{t}^{\beta}\left[\frac{\partial^{2} \psi}{\partial t^{2 \beta}}\right]=\frac{1}{v^{2}} \Psi_{\alpha, \beta}(u, v)-\frac{1}{v^{2}} \Psi_{\alpha}(u, 0)-\frac{1}{v} S_{x}^{\alpha}\left(\frac{\partial^{\beta} \psi\left(x^{\alpha} / \alpha, 0\right)}{\partial t^{\beta}}\right) .
\end{aligned}
$$

The following theorem gives the conformable double Sumudu transform of the fractional partial derivatives $\left(x^{\alpha} / \alpha\right.$ )$\left(\partial^{\beta} \psi / \partial t^{\beta}\right)$ and $\left(x^{\alpha} / \alpha\right)\left(\partial^{2 \beta} \psi / \partial t^{2 \beta}\right)$.

Theorem 10. If conformable double Sumudu transform of the fractional partial derivatives $\left(x^{\alpha} / \alpha\right)\left(\partial^{\beta} \psi / \partial t^{\beta}\right)$ and $\left(x^{\alpha} / \alpha\right)($ $\left.\partial^{2 \beta} \psi / \partial t^{2 \beta}\right)$ are given by

$$
S_{x}^{\alpha} S_{t}^{\beta}\left[\frac{x^{\alpha}}{\alpha} \frac{\partial^{\beta} \psi}{\partial t^{\beta}}\right]=\frac{u}{v} \frac{d}{d u}\left[u \Psi_{\alpha, \beta}(u, v)\right]-\frac{u}{v} \frac{d}{d u}\left[u \Psi_{\alpha}(u, 0)\right]
$$

$$
\begin{aligned}
S_{x}^{\alpha} S_{t}^{\beta}\left[\frac{x^{\alpha}}{\alpha} \frac{\partial^{2} \beta}{\partial t^{2 \beta}}\right]= & \frac{u}{v^{2}} \frac{d}{d u}\left[u \Psi_{\alpha, \beta}(u, v)\right]-\frac{u}{v^{2}} \frac{d}{d u}\left[u \Psi_{\alpha}(u, 0)\right] \\
& -\frac{u}{v} \frac{d}{d u}\left[u S_{x}^{\alpha}\left(\frac{\partial^{\beta} \psi\left(x^{\alpha} / \alpha, 0\right)}{\partial t^{\beta}}\right)\right]
\end{aligned}
$$

respectively. 


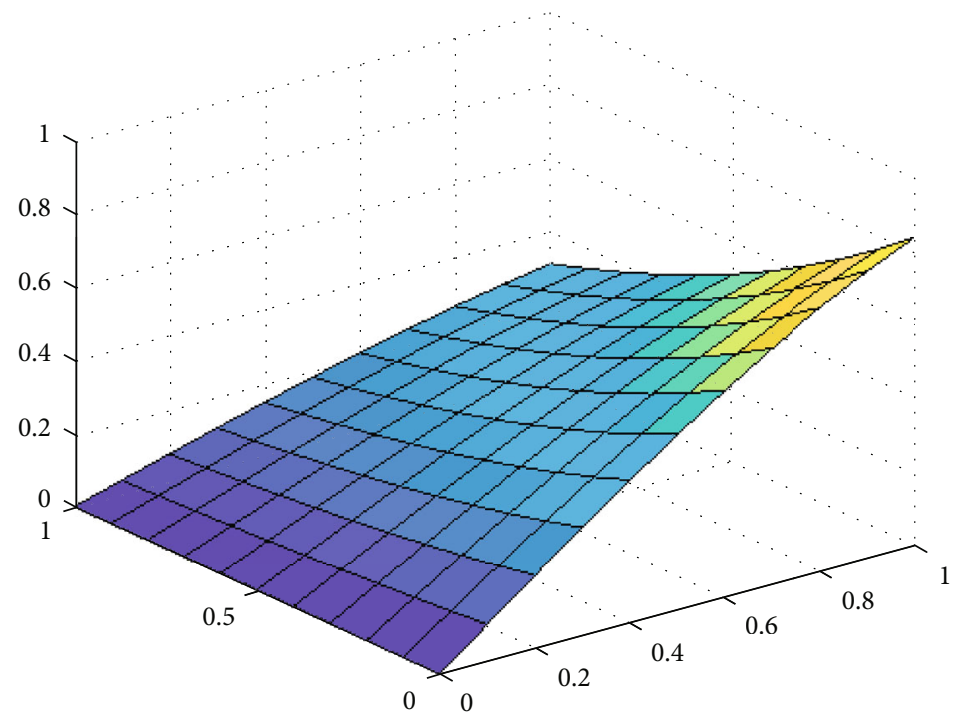

Figure 1: The exact solutions $\psi(x, t)=\varphi(x, t)$ for equation (50) when $\alpha=\beta=1$.

Proof. By using the definition of double Sumudu transform, we have

$$
\begin{aligned}
\frac{d}{d u}\left(S_{x}^{\alpha} S_{t}^{\beta}\left[\frac{\partial^{\beta} \psi}{\partial t^{\beta}}\right]\right) & =\frac{d}{d u} \int_{0}^{\infty} \int_{0}^{\infty} \frac{1}{u v} e^{-\left((1 / u)\left(x^{\alpha} / \alpha\right)+(1 / v)\left(t^{\beta} / \beta\right)\right)} \frac{\partial^{\beta} \psi}{\partial t^{\beta}} x^{\alpha-1} t^{\beta-1} d x d t, \\
& =\int_{0}^{\infty} \frac{1}{v} e^{-(1 / v)\left(t^{\beta} / \beta\right)} \frac{\partial^{\beta} \psi}{\partial t^{\beta}}\left(\int_{0}^{\infty} \frac{d}{d u}\left(\frac{1}{u} e^{-(1 / u)\left(x^{\alpha} / \alpha\right)}\right) x^{\alpha-1} d x\right) t^{\beta-1} d t,
\end{aligned}
$$

we calculate the partial derivative inside brackets as follows:

$$
\begin{aligned}
\int_{0}^{\infty} \frac{d}{d u}\left(\frac{1}{u} e^{-(1 / u)\left(x^{\alpha} / \alpha\right)}\right) x^{\alpha-1} d x & =\int_{0}^{\infty}\left(\frac{1}{u^{3}} \frac{x^{\alpha}}{\alpha}-\frac{1}{u^{2}}\right) e^{-(1 / u)\left(x^{\alpha} / \alpha\right)} x^{\alpha-1} d x \\
= & \int_{0}^{\infty} \frac{1}{u^{3}} \frac{x^{\alpha}}{\alpha} e^{-(1 / u)\left(x^{\alpha} / \alpha\right)} x^{\alpha-1} d x \\
& -\int_{0}^{\infty} \frac{1}{u^{2}} e^{-(1 / u)\left(x^{\alpha} / \alpha\right)} x^{\alpha-1} d x,
\end{aligned}
$$

substituting equation (33) into equation (32), we get

$$
\begin{aligned}
\frac{d}{d u}\left(S_{x}^{\alpha} S_{t}^{\beta}\left[\frac{\partial^{\beta} \psi}{\partial t^{\beta}}\right]\right)= & \int_{0}^{\infty} \frac{1}{v} e^{-(1 / v)\left(t^{\beta} / \beta\right)}\left(\int_{0}^{\infty} \frac{1}{u^{3}} \frac{x^{\alpha}}{\alpha} e^{-(1 / u)\left(x^{\alpha} / \alpha\right)} \frac{\partial^{\beta} \psi}{\partial t^{\beta}} x^{\alpha-1} d x\right) t^{\beta-1} d t \\
& -\int_{0}^{\infty} \frac{1}{v} e^{-(1 / v)\left(t^{\beta} / \beta\right)}\left(\int_{0}^{\infty} \frac{1}{u^{2}} e^{-(1 / u)\left(x^{\alpha} / \alpha\right)} \frac{\partial^{\beta} \psi}{\partial t^{\beta}} x^{\alpha-1} d x\right) t^{\beta-1} d t
\end{aligned}
$$

applying equation (7), we obtain

$$
S_{x}^{\alpha} S_{t}^{\beta}\left[\frac{x^{\alpha}}{\alpha} \frac{\partial^{\beta} \psi}{\partial t^{\beta}}\right]=u^{2} \frac{d}{d u} S_{x}^{\alpha} S_{t}^{\beta}\left[\frac{\partial^{\beta} \psi}{\partial t^{\beta}}\right]+u S_{x}^{\alpha} S_{t}^{\beta}\left[\frac{\partial^{\beta} \psi}{\partial t^{\beta}}\right],
$$

and on using equation (28), we have

$$
S_{x}^{\alpha} S_{t}^{\beta}\left[\frac{x^{\alpha}}{\alpha} \frac{\partial^{\beta} \psi}{\partial t^{\beta}}\right]=\frac{u}{v} \frac{d}{d u}\left[u \Psi_{\alpha, \beta}(u, v)\right]-\frac{u}{v} \frac{d}{d u}\left[u \Psi_{\alpha}(u, 0)\right] .
$$

In a similar manner, one can prove equation (31).

\section{One-Dimensional Fractional Coupled Burgers' Equation}

In this section, the solution of regular and irregular onedimensional conformable fractional coupled burgers' equations is discussed by using conformable double Sumudu decomposition methods (CDSDM). By taking $\alpha=1$ and $\beta$ $=1$ in the following problems, one can get the problems which were discussed in [21]:

The first problem is as follows: we consider the following one-dimensional conformable fractional coupled burgers' equations:

$$
\begin{aligned}
& \frac{\partial^{\beta} \psi}{\partial t^{\beta}}-\frac{\partial^{2 \alpha} \psi}{\partial x^{2 \alpha}}+\eta \psi \frac{\partial^{\alpha} \psi}{\partial x^{\alpha}}+\zeta \frac{\partial^{\alpha}}{\partial x^{\alpha}}(\psi \varphi)=f\left(\frac{x^{\alpha}}{\alpha}, \frac{t^{\beta}}{\beta}\right), \\
& \frac{\partial^{\beta} \varphi}{\partial t^{\beta}}-\frac{\partial^{2 \alpha} \varphi}{\partial x^{2 \alpha}}+\eta v \frac{\partial^{\alpha} \varphi}{\partial x^{\alpha}}+\mu \frac{\partial^{\alpha}}{\partial x^{\alpha}}(\psi \varphi)=g\left(\frac{x^{\alpha}}{\alpha}, \frac{t^{\beta}}{\beta}\right),
\end{aligned}
$$

appropriate initial conditions

$$
\psi\left(\frac{x^{\alpha}}{\alpha}, 0\right)=f_{1}\left(\frac{x^{\alpha}}{\alpha}\right), \varphi\left(\frac{x^{\alpha}}{\alpha}, 0\right)=g_{1}\left(\frac{x^{\alpha}}{\alpha}\right),
$$

where $f\left(x^{\alpha} / \alpha, t^{\beta} / \beta\right), g\left(x^{\alpha} / \alpha, t^{\beta} / \beta\right), f_{1}\left(x^{\alpha} / \alpha\right)$, and $g_{1}\left(x^{\alpha} / \alpha\right)$ are known functions and $\eta, \zeta$, and $\mu$ are arbitrary constants depending on the system parameters. Employing the conformable double Sumudu transform for both sides of 


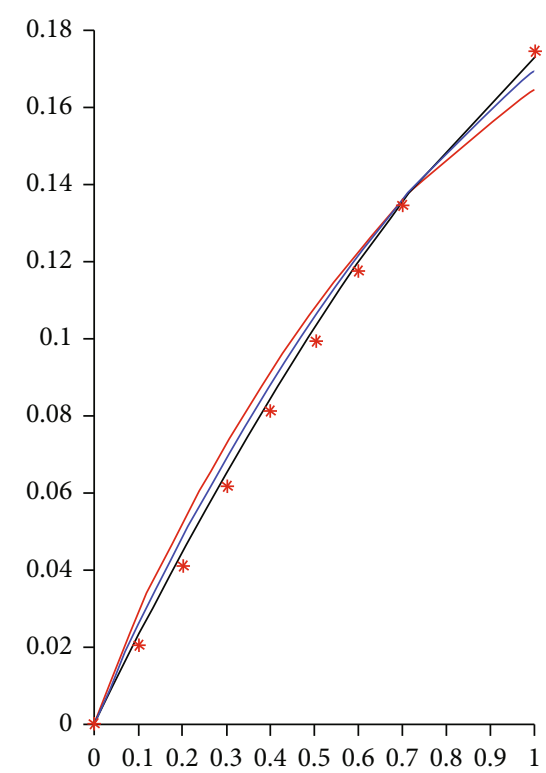

* Exact solution

- Alpha $=$ beta $=0.85$ and $t=1$

_ Alpha $=$ beta $=0.90$ and $t=1$

- Alpha $=$ beta $=0.95$ and $t=1$

(a) The solutions $\psi(x, t)=\varphi(x, t)$ for equation (50) at $\alpha=\beta=1, \alpha=\beta=0.85, \alpha=\beta=0.90$, and $\alpha=\beta=0.95$

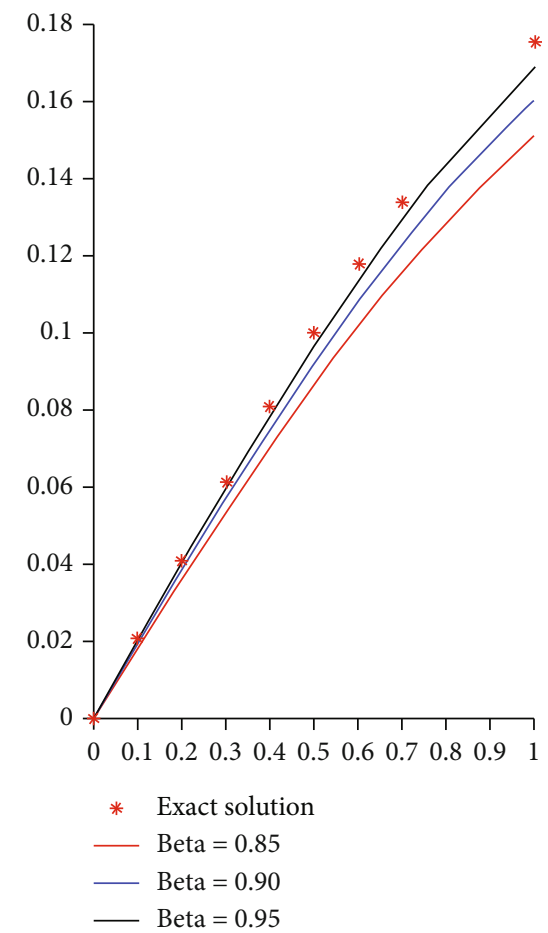

(b) The solutions $\psi(x, t)=\varphi(x, t)$ for equation (50) when $\alpha=0.99$ and different values of $\beta$

Figure 2: Continued. 


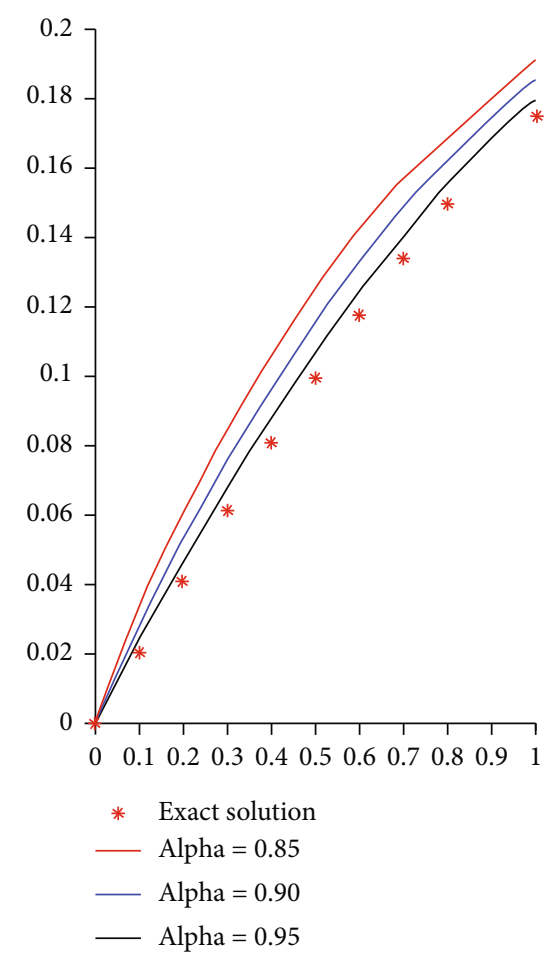

(c) The solutions $\psi(x, t)=\varphi(x, t)$ for equation (50) for different values of $\alpha$ at $\beta=0.99$

Figure 2: The solutions $\psi(x, t)=\varphi(x, t)$ for equation (50) at different values of $\alpha$ and $\beta t=\pi / 2$.

equation (37) and conformable single Sumudu transform for equation (38), we obtain

$\Psi_{\alpha, \beta}(u, v)=\Psi_{\alpha}(u, 0)+v S_{x}^{\alpha} S_{\mathrm{t}}^{\beta}\left[f\left(\frac{x^{\alpha}}{\alpha}, \frac{t^{\beta}}{\beta}\right)+\frac{\partial^{2 \alpha} \psi}{\partial x^{2 \alpha}}-\eta \psi \frac{\partial^{\alpha} \psi}{\partial x^{\alpha}}-\zeta \frac{\partial^{\alpha}}{\partial x^{\alpha}}(\psi \varphi)\right]$,

$\Phi_{\alpha, \beta}(u, v)=\Phi_{\alpha}(u, 0)+v S_{x}^{\alpha} S_{t}^{\beta}\left[g\left(\frac{x^{\alpha}}{\alpha}, \frac{t^{\beta}}{\beta}\right)+\frac{\partial^{2 \alpha} \varphi}{\partial x^{2 \alpha}}-\eta \varphi \frac{\partial^{\alpha} \varphi}{\partial x^{\alpha}}-\zeta \frac{\partial^{\alpha}}{\partial x^{\alpha}}(\psi \varphi)\right]$.

The solution of one-dimensional conformable fractional coupled burgers' equations is defined by the infinite series as follows:

$$
\begin{aligned}
& \psi\left(\frac{x^{\alpha}}{\alpha}, \frac{t^{\beta}}{\beta}\right)=\sum_{n=0}^{v} \varphi_{n}\left(\frac{x^{\alpha}}{\alpha}, \frac{t^{\beta}}{\beta}\right), \\
& \varphi\left(\frac{x^{\alpha}}{\alpha}, \frac{t^{\beta}}{\beta}\right)=\sum_{n=0}^{v} \varphi_{n}\left(\frac{x^{\alpha}}{\alpha}, \frac{t^{\beta}}{\beta}\right) .
\end{aligned}
$$

The nonlinear operators $A_{n}, B_{n}$, and $C_{n}$ defined by Ado- mian polynomials are given as follows:

$$
\begin{aligned}
& A_{n}={ }_{n=0}^{\infty} \psi_{n} \psi_{x n}, \\
& B_{n}=\sum_{n=0}^{v} \varphi_{n} \varphi_{x n}, \\
& C_{n}=\sum_{n=0}^{v} \psi_{n} \varphi_{n} .
\end{aligned}
$$

The few components of the Adomian polynomials are given as follows:

$$
\begin{gathered}
A_{0}=\psi_{0} \psi_{0 x}, \\
A_{1}=\psi_{0} \psi_{1 x}+\psi_{1} \psi_{0 x}, \\
A_{2}=\psi_{0} \psi_{2 x}+\psi_{1} \psi_{1 x}+\psi_{2} \psi_{0 x}, \\
A_{3}=\psi_{0} \psi_{3 x}+\psi_{1} \psi_{1 x}+\psi_{2} \psi_{1 x}+\psi_{3} \psi_{0 x}, \\
A_{4}=\psi_{0} \psi_{4 x}+\psi_{1} \psi_{3 x}+\psi_{2} \psi_{2 x}+\psi_{3} \psi_{1 x}+\psi_{4} \psi_{0 x}, \\
B_{0}=\varphi_{0} \varphi_{0 x}, \\
B_{1}=\varphi_{0} \varphi_{1 x}+\varphi_{1} \varphi_{0 x}, \\
B_{2}=\varphi_{0} \varphi_{2 x}+\varphi_{1} \varphi_{1 x}+\varphi_{2} \varphi_{0 x}, \\
B_{3}=\varphi_{0} \varphi_{3 x}+\varphi_{1} \varphi_{2 x}+\varphi_{2} \varphi_{1 x}+\varphi_{3} \varphi_{0 x}, \\
B_{4}=\varphi_{0} \varphi_{4 x}+\varphi_{1} \varphi_{3 x}+\varphi_{2} \varphi_{2 x}+\varphi_{3} \varphi_{1 x}+\varphi_{4} \varphi_{0 x},
\end{gathered}
$$




$$
\begin{gathered}
C_{0}=\psi_{0} \varphi_{0}, \\
C_{1}=\psi_{0} \varphi_{0}+\psi_{1} \varphi_{0} \\
C_{2}=\psi_{0} \varphi_{2}+\psi_{1} \varphi_{1}+\psi_{2} \varphi_{0} . \\
C_{3}=\psi_{0} \varphi_{3}+\psi_{1} \varphi_{2}+\psi_{2} \varphi_{1}+\psi_{3} \varphi_{0}, \\
C_{3}=\psi_{0} \varphi_{4}+\psi_{1} \varphi_{3}+\psi_{2} \varphi_{2}+\psi_{3} \varphi_{1}+\psi_{4} \varphi_{0} .
\end{gathered}
$$

Applying the inverse double Sumudu transform to equation (39), equation (40) and using equation (42), we get

$$
\begin{aligned}
\sum_{n=0}^{\infty} \psi_{n}\left(\frac{x^{\alpha}}{\alpha}, \frac{t^{\beta}}{\beta}\right)= & f_{1}\left(\frac{x^{\alpha}}{\alpha}\right)+S_{u}^{-1} S_{v}^{-1}\left[v S_{x}^{\alpha} S_{t}^{\beta}\left[\frac{\partial^{2 \alpha} \psi_{n}}{\partial x^{2 \alpha}}\right]\right] \\
& -S_{u}^{-1} S_{v}^{-1}\left[v S_{x}^{\alpha} S_{t}^{\beta}\left[\eta A_{n}\right]\right]-S_{u}^{-1} S_{v}^{-1}\left[v S_{x}^{\alpha} S_{t}^{\beta}\left[\zeta\left(C_{n}\right)\right]\right], \\
\sum_{n=0}^{\infty} \varphi_{n}\left(\frac{x^{\alpha}}{\alpha}, \frac{t^{\beta}}{\beta}\right)= & g_{1}\left(\frac{x^{\alpha}}{\alpha}\right)+S_{u}^{-1} S_{v}^{-1}[v G(u, v)]+S_{u}^{-1} S_{v}^{-1} \\
& \cdot\left[v S_{x}^{\alpha} S_{t}^{\beta}\left[\frac{\partial^{2 \alpha} \varphi_{n}}{\partial x^{2 \alpha}}\right]\right]-S_{u}^{-1} S_{v}^{-1}\left[v S_{x}^{\alpha} S_{t}^{\beta}\left[\eta B_{n}\right]\right] \\
& -S_{u}^{-1} S_{v}^{-1}\left[v S_{x}^{\alpha} S_{t}^{\beta}\left[\mu\left(C_{n}\right)\right]\right] .
\end{aligned}
$$

For the case $n=0$, we set

$$
\begin{aligned}
& \psi_{0}=f_{1}\left(\frac{x^{\alpha}}{\alpha}\right)+S_{u}^{-1} S_{v}^{-1}[v F(u, v)], \\
& \varphi_{0}=g_{1}\left(\frac{x^{\alpha}}{\alpha}\right)+S_{u}^{-1} S_{v}^{-1}[v G(u, v)] .
\end{aligned}
$$

Now, we can obtain the following general form:

$\psi_{n+1}=S_{u}^{-1} S_{v}^{-1}\left[v S_{x}^{\alpha} S_{t}^{\beta}\left[\frac{\partial^{2 \alpha} \psi_{n}}{\partial x^{2 \alpha}}\right]\right]-S_{u}^{-1} S_{v}^{-1}\left[v S_{x}^{\alpha} S_{t}^{\beta}\left[\eta A_{n}\right]\right]-S_{u}^{-1} S_{v}^{-1}\left[v S_{x}^{\alpha} S_{t}^{\beta}\left[\zeta\left(C_{n}\right)\right]\right]$,

$$
\begin{aligned}
\varphi_{n+1}= & S_{u}^{-1} S_{v}^{-1}\left[v S_{x}^{\alpha} S_{t}^{\beta}\left[\frac{\partial^{2 \alpha} \varphi_{n}}{\partial x^{2 \alpha}}\right]\right]-S_{u}^{-1} S_{v}^{-1}\left[v S_{x}^{\alpha} S_{t}^{\beta}\left[\eta B_{n}\right]\right] \\
& -S_{u}^{-1} S_{v}^{-1}\left[v S_{x}^{\alpha} S_{t}^{\beta}\left[\mu\left(C_{n}\right)\right]\right] .
\end{aligned}
$$

Here, we provide double inverse Sumudu transform with respect to $u$ and $v$ existing for each term in the right-hand side of the above equations. To show the method of coupled one-dimensional conformable fractional coupled burgers' equations, the following example is considered.

Example 11. Consider the following homogeneous form of a one-dimensional conformable fractional coupled burgers' equations:

$$
\begin{aligned}
& \frac{\partial^{\beta} \psi}{\partial t^{\beta}}-\frac{\partial^{2 \alpha} \psi}{\partial x^{2 \alpha}}-2 \psi \frac{\partial^{\alpha} \psi}{\partial x^{\alpha}}+\frac{\partial^{\alpha}}{\partial x^{\alpha}}(\psi \varphi)=0, \\
& \frac{\partial^{\beta} \varphi}{\partial t^{\beta}}-\frac{\partial^{2 \alpha} \varphi}{\partial x^{2 \alpha}}-2 \varphi \frac{\partial^{\alpha} \varphi}{\partial x^{\alpha}}+\frac{\partial^{\alpha}}{\partial x^{\alpha}}(\psi \varphi)=0,
\end{aligned}
$$

with initial condition

$$
\psi\left(\frac{x^{\alpha}}{\alpha}, 0\right)=\sin \frac{x^{\alpha}}{\alpha}, \varphi\left(\frac{x^{\alpha}}{\alpha}, 0\right)=\sin \frac{x^{\alpha}}{\alpha}
$$

According to equations (47), (48), and (49), the first three terms of the Sumudu decomposition series are derived as follows:

$$
\begin{aligned}
& \psi_{0}=\sin \frac{x^{\alpha}}{\alpha}, \varphi_{0}=\sin \frac{x^{\alpha}}{\alpha} \mathrm{red} \\
& \psi_{1}=S_{u}^{-1} S_{v}^{-1}\left[v S_{x}^{\alpha} S_{t}^{\beta}\left[\frac{\partial^{2 \alpha} \psi_{0}}{\partial x^{2 \alpha}}+2 \psi_{0} \frac{\partial^{\alpha} \psi_{0}}{\partial x^{\alpha}}-\frac{\partial^{\alpha}}{\partial x^{\alpha}}\left(\psi_{0} \varphi_{0}\right)\right]\right] \\
& =S_{u}^{-1} S_{v}^{-1}\left[v S_{x}^{\alpha} S_{t}^{\beta}\left[-\sin \frac{x^{\alpha}}{\alpha}\right]\right]=S_{u}^{-1} S_{v}^{-1}\left[\frac{u v}{\left(1+u^{2}\right)}\right]=-\frac{t^{\beta}}{\beta} \sin \frac{x^{\alpha}}{\alpha}, \\
& \varphi_{1}=S_{u}^{-1} S_{v}^{-1}\left[v S_{x}^{\alpha} S_{t}^{\beta}\left[\frac{\partial^{2 \alpha} \varphi_{0}}{\partial x^{2 \alpha}}+2 \varphi_{0} \frac{\partial^{\alpha} \varphi_{0}}{\partial x^{\alpha}}-\frac{\partial^{\alpha}}{\partial x^{\alpha}}\left(\psi_{0} \varphi_{0}\right)\right]\right] \\
& =S_{u}^{-1} S_{v}^{-1}\left[v S_{x}^{\alpha} S_{t}^{\beta}\left[-\sin \frac{x^{\alpha}}{\alpha}\right]\right]=S_{u}^{-1} S_{v}^{-1}\left[\frac{u v}{\left(u^{2}+1\right)}\right]=-\frac{t^{\beta}}{\beta} \sin \frac{x^{\alpha}}{\alpha} \mathrm{red}, \\
& \psi_{2}=S_{u}^{-1} S_{v}^{-1}\left[v S _ { x } ^ { \alpha } S _ { t } ^ { \beta } \left[\frac{\partial^{2 \alpha} \psi_{1}}{\partial x^{2 \alpha}}+2\left(\psi_{0} \frac{\partial^{\alpha}}{\partial x^{\alpha}} \psi_{1}+\psi_{1} \frac{\partial^{\alpha}}{\partial x^{\alpha}} \psi_{0}\right)\right.\right. \\
& \left.\left.-\frac{\partial^{\alpha}}{\partial x^{\alpha}}\left(\psi_{0} \varphi_{1}+\psi_{1} \varphi_{0}\right)\right]\right]=S_{u}^{-1} S_{v}^{-1}\left[v S_{x}^{\alpha} S_{t}^{\beta}\left[\frac{t^{\beta}}{\beta} \sin \frac{x^{\alpha}}{\alpha}\right]\right] \\
& =S_{u}^{-1} S_{v}^{-1}\left[\frac{u v^{2}}{\left(u^{2}+1\right)}\right]=\frac{\left(t^{\beta} / \beta\right)^{2}}{2} \sin \frac{x^{\alpha}}{\alpha} \text {, } \\
& \varphi_{2}=S_{u}^{-1} S_{v}^{-1}\left[v S _ { x } ^ { \alpha } S _ { t } ^ { \beta } \left[\frac{\partial^{2 \alpha} \varphi_{1}}{\partial x^{2 \alpha}}+2\left(\varphi_{0} \frac{\partial^{\alpha}}{\partial x^{\alpha}} \varphi_{1}+\varphi_{1} \frac{\partial^{\alpha}}{\partial x^{\alpha}} \varphi_{0}\right)\right.\right. \\
& \left.\left.-\frac{\partial^{\alpha}}{\partial x^{\alpha}}\left(\psi_{0} \varphi_{1}+\psi_{1} \varphi_{0}\right)\right]\right]=S_{u}^{-1} S_{v}^{-1}\left[v S_{x}^{\alpha} S_{t}^{\beta}\left[\frac{t^{\beta}}{\beta} \sin \frac{x^{\alpha}}{\alpha}\right]\right] \\
& =S_{u}^{-1} S_{v}^{-1}\left[\frac{u v^{2}}{\left(u^{2}+1\right)}\right]=\frac{\left(t^{\beta} / \beta\right)^{2}}{2} \sin \frac{x^{\alpha}}{\alpha}, \\
& \psi_{3}=S_{u}^{-1} S_{v}^{-1}\left[v S_{x}^{\alpha} S_{t}^{\beta}\left[\frac{\partial^{2 \alpha} \psi_{2}}{\partial x^{2 \alpha}}+2\left(\psi_{0} \frac{\partial^{\alpha}}{\partial x^{\alpha}} \psi_{2}+\psi_{1} \frac{\partial^{\alpha}}{\partial x^{\alpha}} \psi_{1}+\psi_{2} \frac{\partial^{\alpha}}{\partial x^{\alpha}} \psi_{0}\right)\right]\right] \\
& -S_{u}^{-1} S_{v}^{-1}\left[v S_{x}^{\alpha} S_{t}^{\beta}\left[\frac{\partial^{\alpha}}{\partial x^{\alpha}}\left(\psi_{0} \varphi_{2}+\psi_{1} \varphi_{1}+\psi_{2} \varphi_{0}\right)\right]\right]=-\frac{\left(t^{\beta} / \beta\right)^{3}}{6} \sin \frac{x^{\alpha}}{\alpha},
\end{aligned}
$$




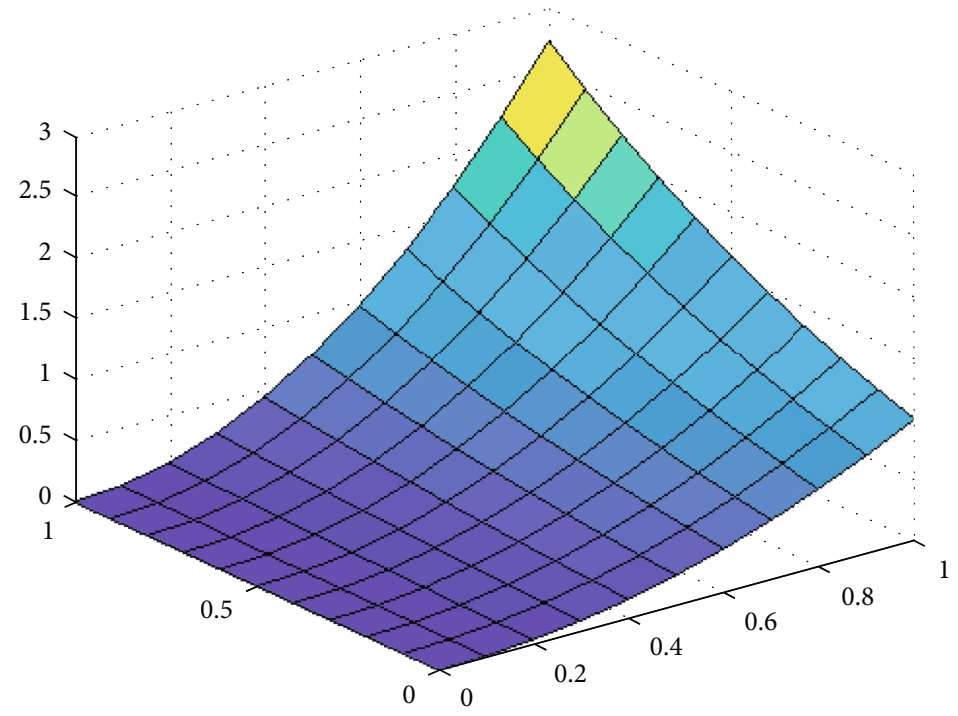

Figure 3: The solutions $\psi(x, t)=\varphi(x, t)$ for equation (68) when $\alpha=\beta=1$.

$$
\begin{aligned}
\varphi_{3}= & S_{u}^{-1} S_{v}^{-1}\left[v S_{x}^{\alpha} S_{t}^{\beta}\left[\frac{\partial^{2 \alpha} \varphi_{2}}{\partial x^{2 \alpha}}+2\left(\varphi_{0} \frac{\partial^{\alpha}}{\partial x^{\alpha}} \varphi_{2}+\varphi_{1} \frac{\partial^{\alpha}}{\partial x^{\alpha}} \varphi_{1}+\varphi_{2} \frac{\partial^{\alpha}}{\partial x^{\alpha}} \varphi_{0}\right)\right]\right] \\
& -S_{u}^{-1} S_{v}^{-1}\left[v S_{x}^{\alpha} S_{t}^{\beta}\left[\frac{\partial^{\alpha}}{\partial x^{\alpha}}\left(\psi_{0} \varphi_{2}+\psi_{1} \varphi_{1}+\psi_{2} \varphi_{0}\right)\right]\right]=-\frac{\left(t^{\beta} / \beta\right)^{3}}{6} \sin \frac{x^{\alpha}}{\alpha},
\end{aligned}
$$

and so on for other components. Using equation (41), the series solutions are therefore given by

$$
\begin{aligned}
& \psi\left(\frac{x^{\alpha}}{\alpha}, \frac{t^{\beta}}{\beta}\right)=\psi_{0}+\psi_{2}+\psi_{3}+\cdots=\left(1-\left(\frac{t^{\beta}}{\beta}\right)+\frac{\left(t^{\beta} / \beta\right)^{2}}{2 !}-\frac{\left(t^{\beta} / \beta\right)^{3}}{3 !}+\cdots\right) \sin \frac{x^{\alpha}}{\alpha}, \\
& \varphi\left(\frac{x^{\alpha}}{\alpha}, \frac{t^{\beta}}{\beta}\right)=\varphi_{0}+\varphi_{2}+\varphi_{3}+\cdots=\left(1-\left(\frac{t^{\beta}}{\beta}\right)+\frac{\left(t^{\beta} / \beta\right)^{2}}{2 !}-\frac{\left(t^{\beta} / \beta\right)^{3}}{3 !}+\cdots\right) \sin \frac{x^{\alpha}}{\alpha} .
\end{aligned}
$$

Therefore, the exact solution takes the form of

$$
\begin{aligned}
& \psi\left(\frac{x^{\alpha}}{\alpha}, \frac{t^{\beta}}{\beta}\right)=e^{-t^{\beta} / \beta} \sin \frac{x^{\alpha}}{\alpha}, \\
& \varphi\left(\frac{x^{\alpha}}{\alpha}, \frac{t^{\beta}}{\beta}\right)=e^{-t^{\beta} / \beta} \sin \frac{x^{\alpha}}{\alpha} .
\end{aligned}
$$

By taking $\alpha=1$ and $\beta=1$, the fractional solution become

$$
\begin{aligned}
& \psi(x, t)=e^{-t} \sin x, \\
& \varphi(x, t)=e^{-t} \sin x .
\end{aligned}
$$

The exact solution of equation (50) for $\alpha=\beta=1$ is shown in Figure 1 (see also Figures 2(a)-2(c)) for $\alpha \neq \beta$.

The second problem is as follows: we consider the singular one-dimensional conformable fractional coupled burgers' equations of the form:

$$
\begin{aligned}
& \frac{\partial^{\beta} \psi}{\partial t^{\beta}}-\frac{\alpha}{x^{\alpha}} \frac{\partial^{\alpha}}{\partial x^{\alpha}}\left(\frac{x^{\alpha}}{\alpha} \frac{\partial^{\alpha} \psi}{\partial x^{\alpha}}\right)+\eta \psi \frac{\partial^{\alpha} \psi}{\partial x^{\alpha}}+\zeta \frac{\partial^{\alpha}}{\partial x^{\alpha}}(\psi \varphi)=f\left(\frac{x^{\alpha}}{\alpha}, \frac{t^{\beta}}{\beta}\right), \\
& \frac{\partial^{\beta} \varphi}{\partial t^{\beta}}-\frac{\alpha}{x^{\alpha}} \frac{\partial^{\alpha}}{\partial x^{\alpha}}\left(\frac{x^{\alpha}}{\alpha} \frac{\partial^{\alpha} \varphi}{\partial x^{\alpha}}\right)+\eta \psi \frac{\partial^{\alpha} \varphi}{\partial x^{\alpha}}+\mu \frac{\partial^{\alpha}}{\partial x^{\alpha}}(\psi \varphi)=g\left(\frac{x^{\alpha}}{\alpha}, \frac{t^{\beta}}{\beta}\right),
\end{aligned}
$$

with initial conditions

$$
\psi\left(\frac{x^{\alpha}}{\alpha}, 0\right)=f_{1}\left(\frac{x^{\alpha}}{\alpha}\right), \quad \varphi\left(\frac{x^{\alpha}}{\alpha}, 0\right)=g_{1}\left(\frac{x^{\alpha}}{\alpha}\right),
$$

where $\left(\alpha / x^{\alpha}\right)\left(\partial^{\alpha} / \partial x^{\alpha}\right)\left(\left(x^{\alpha} / \alpha\right)\left(\partial^{\alpha} / \partial x^{\alpha}\right)\right)$ is a conformable Bessel operator and $\zeta, \mu$, and $\eta$ are real constants. In order to obtain the solution of equation (56). First, multiplying equation (56) by $x^{\alpha} / \alpha$ to obtain the following:

$$
\begin{aligned}
& \frac{x^{\alpha}}{\alpha} \frac{\partial^{\beta} \psi}{\partial t^{\beta}}-\frac{\partial^{\alpha}}{\partial x^{\alpha}}\left(\frac{x^{\alpha}}{\alpha} \frac{\partial^{\alpha} \psi}{\partial x^{\alpha}}\right)+\eta \frac{x^{\alpha}}{\alpha} \psi \frac{\partial^{\alpha} \psi}{\partial x^{\alpha}}+\zeta \frac{x^{\alpha}}{\alpha} \frac{\partial^{\alpha}}{\partial x^{\alpha}}(\psi \varphi)=\frac{x^{\alpha}}{\alpha} f\left(\frac{x^{\alpha}}{\alpha}, \frac{t^{\beta}}{\beta}\right), \\
& \frac{x^{\alpha}}{\alpha} \frac{\partial^{\beta} \varphi}{\partial t^{\beta}}-\frac{\partial^{\alpha}}{\partial x^{\alpha}}\left(\frac{x^{\alpha}}{\alpha} \frac{\partial^{\alpha} \varphi}{\partial x^{\alpha}}\right)+\eta \frac{x^{\alpha}}{\alpha} \varphi \frac{\partial^{\alpha} \varphi}{\partial x^{\alpha}}+\mu \frac{x^{\alpha}}{\alpha} \frac{\partial^{\alpha}}{\partial x^{\alpha}}(\psi \varphi)=\frac{x^{\alpha}}{\alpha} g\left(\frac{x^{\alpha}}{\alpha}, \frac{t^{\beta}}{\beta}\right) .
\end{aligned}
$$

Second, applying the conformable double Sumudu transform on both sides of equation (58) and after using the differentiation property of conformable Sumudu transform, we get

$$
\begin{aligned}
& S_{x}^{\alpha} S_{t}^{\beta}\left[\frac{x^{\alpha}}{\alpha} \frac{\partial^{\beta} \psi}{\partial t^{\beta}}\right]=S_{x}^{\alpha} S_{t}^{\beta}\left[\frac{\partial^{\alpha}}{\partial x^{\alpha}}\left(\frac{x^{\alpha}}{\alpha} \frac{\partial^{\alpha} \psi}{\partial x^{\alpha}}\right)-\eta \frac{x^{\alpha}}{\alpha} \psi \frac{\partial^{\alpha} \psi}{\partial x^{\alpha}}-\zeta \frac{x^{\alpha}}{\alpha} \frac{\partial^{\alpha}}{\partial x^{\alpha}}(\psi \varphi)+\frac{x^{\alpha}}{\alpha} f\left(\frac{x^{\alpha}}{\alpha}, \frac{t^{\beta}}{\beta}\right)\right], \\
& S_{x}^{\alpha} S_{t}^{\beta}\left[\frac{x^{\alpha}}{\alpha} \frac{\partial^{\beta} \varphi}{\partial t^{\beta}}\right]=S_{x}^{\alpha} S_{t}^{\beta}\left[\frac{\partial^{\alpha}}{\partial x^{\alpha}}\left(\frac{x^{\alpha}}{\alpha} \frac{\partial^{\alpha} \varphi}{\partial x^{\alpha}}\right)-\eta \frac{x^{\alpha}}{\alpha} \varphi \frac{\partial^{\alpha} \varphi}{\partial x^{\alpha}}-\mu \frac{x^{\alpha}}{\alpha} \frac{\partial^{\alpha}}{\partial x^{\alpha}}(\psi \varphi)+\frac{x^{\alpha}}{\alpha} g\left(\frac{x^{\alpha}}{\alpha}, \frac{t^{\beta}}{\beta}\right)\right] .
\end{aligned}
$$




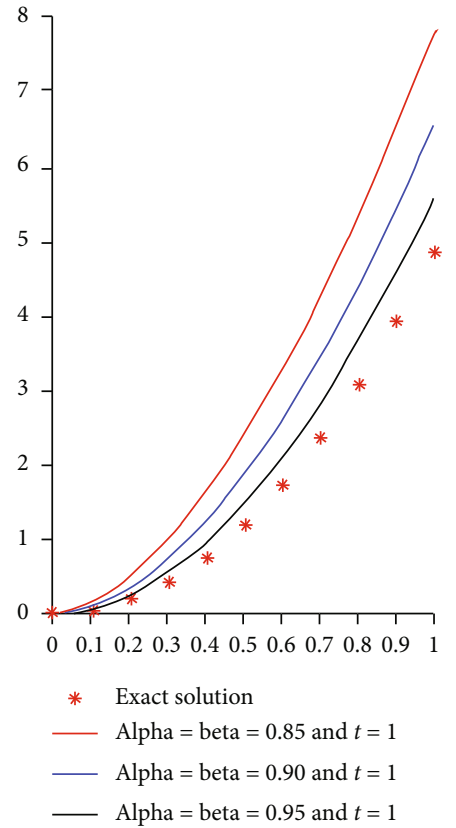

(a) The solution $\psi(x, t)=\varphi(x, t)$ for (68) at $\alpha=\beta$

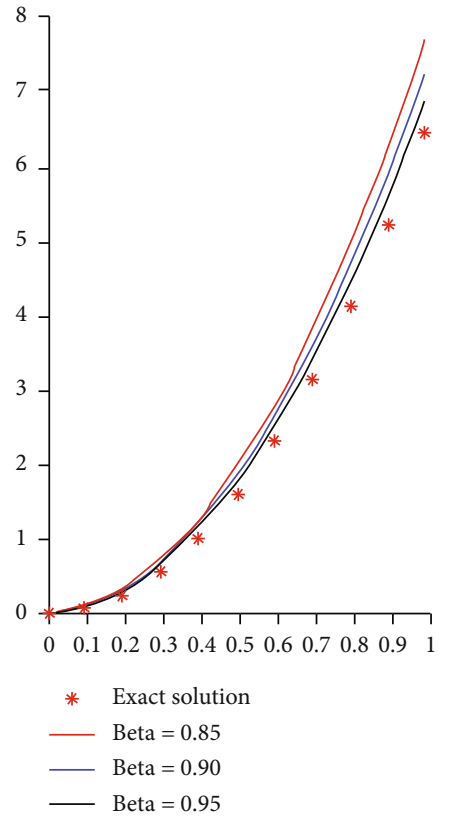

(b) The solution $\psi(x, t)=\varphi(x, t)$ for (68) when $\alpha=0.99$ and different values of $\beta$

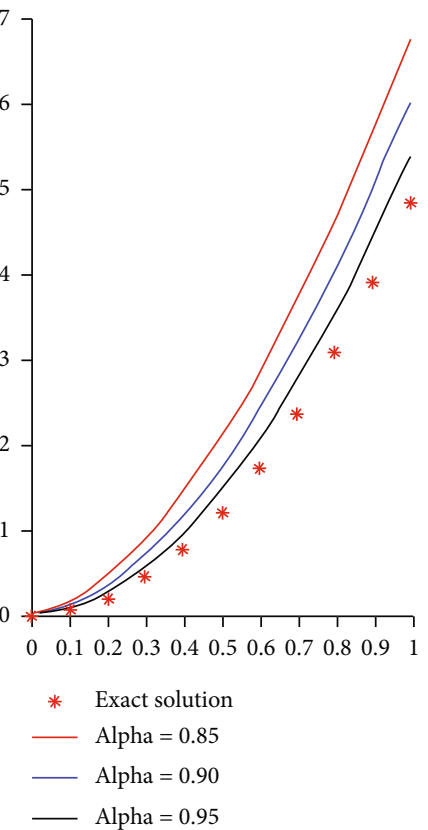

(c) The solution $\psi(x, t)=\varphi(x, t)$ for (68) for different values of $\alpha$ at $\beta=0.99$
On using Theorems 9 and 10, we have

$$
\begin{aligned}
& \frac{u}{v} \frac{d}{d u}\left[u \Psi_{\alpha, \beta}(u, v)\right]-\frac{u}{v} \frac{d}{d u}\left[u \Psi_{\alpha}(u, 0)\right] \\
& \quad=u^{2} \frac{\partial F_{\alpha, \beta}(u, v)}{\partial u}+u F_{\alpha, \beta}(u, v)++S_{x}^{\alpha} S_{t}^{\beta} \\
& \cdot\left[\frac{\partial^{\alpha}}{\partial x^{\alpha}}\left(\frac{x^{\alpha}}{\alpha} \frac{\partial^{\alpha} \psi}{\partial x^{\alpha}}\right)-\eta \frac{x^{\alpha}}{\alpha} \psi \frac{\partial^{\alpha} \psi}{\partial x^{\alpha}}-\zeta \frac{x^{\alpha}}{\alpha} \frac{\partial^{\alpha}}{\partial x^{\alpha}}(\psi \varphi)\right],
\end{aligned}
$$

$$
\begin{aligned}
& \frac{u}{v} \frac{d}{d u}\left[u \Phi_{\alpha, \beta}(u, v)\right]-\frac{u}{v} \frac{d}{d u}\left[u \Phi_{\alpha}(u, 0)\right] \\
& \quad=u^{2} \frac{\partial G_{\alpha, \beta}(u, v)}{\partial u}+u G_{\alpha, \beta}(u, v)+S_{x}^{\alpha} S_{t}^{\beta} \\
& \cdot\left[\frac{\partial^{\alpha}}{\partial x^{\alpha}}\left(\frac{x^{\alpha}}{\alpha} \frac{\partial^{\alpha} \varphi}{\partial x^{\alpha}}\right)-\eta \frac{x^{\alpha}}{\alpha} \varphi \frac{\partial^{\alpha} \varphi}{\partial x^{\alpha}}-\mu \frac{x^{\alpha}}{\alpha} \frac{\partial^{\alpha}}{\partial x^{\alpha}}(\psi \varphi)\right],
\end{aligned}
$$

and arranging equation (60), we obtain 


$$
\begin{aligned}
\frac{d}{d u}\left[u \Psi_{\alpha, \beta}(u, v)\right]= & u v \frac{\partial F_{\alpha, \beta}(u, v)}{\partial u}+v F_{\alpha, \beta}(u, v)+\frac{d}{d u}\left[u \Psi_{\alpha}(u, 0)\right]+\frac{v}{u} S_{x}^{\alpha} S_{t}^{\beta} \\
& \cdot\left[\frac{\partial^{\alpha}}{\partial x^{\alpha}}\left(\frac{x^{\alpha}}{\alpha} \frac{\partial^{\alpha} \psi}{\partial x^{\alpha}}\right)-\eta \frac{x^{\alpha}}{\alpha} \psi \frac{\partial^{\alpha} \psi}{\partial x^{\alpha}}-\zeta \frac{x^{\alpha}}{\alpha} \frac{\partial^{\alpha}}{\partial x^{\alpha}}(\psi \varphi)\right], \\
\frac{d}{d u}\left[u \Phi_{\alpha, \beta}(u, v)\right]= & u v \frac{\partial G_{\alpha, \beta}(u, v)}{\partial u}+v G_{\alpha, \beta}(u, v)+\frac{d}{d u}\left[u \Phi_{\alpha}(u, 0)\right]+\frac{v}{u} S_{x}^{\alpha} S_{t}^{\beta} \\
& \cdot\left[\frac{\partial^{\alpha}}{\partial x^{\alpha}}\left(\frac{x^{\alpha}}{\alpha} \frac{\partial^{\alpha} \varphi}{\partial x^{\alpha}}\right)-\eta \frac{x^{\alpha}}{\alpha} \varphi \frac{\partial^{\alpha} \varphi}{\partial x^{\alpha}}-\mu \frac{x^{\alpha}}{\alpha} \frac{\partial^{\alpha}}{\partial x^{\alpha}}(\psi \varphi)\right] .
\end{aligned}
$$

Third, by applying the integral for both sides of equation (61) from 0 to $u$ with respect to $u$, we have

$$
\begin{aligned}
\Psi_{\alpha, \beta}(u, v)= & \frac{1}{u} \int_{0}^{u}\left(u v \frac{\partial F_{\alpha, \beta}(u, v)}{\partial u}+v F_{\alpha, \beta}(u, v)+\frac{d}{d u}\left[u \Psi_{\alpha}(u, 0)\right]\right) d u \\
& +\frac{1}{u} \int_{0}^{u} \frac{v}{u} S_{x}^{\alpha} S_{t}^{\beta}\left[\frac{\partial^{\alpha}}{\partial x^{\alpha}}\left(\frac{x^{\alpha}}{\alpha} \frac{\partial^{\alpha} \psi}{\partial x^{\alpha}}\right)-\eta \frac{x^{\alpha}}{\alpha} \psi \frac{\partial^{\alpha} \psi}{\partial x^{\alpha}}-\zeta \frac{x^{\alpha}}{\alpha} \frac{\partial^{\alpha}}{\partial x^{\alpha}}(\psi \varphi)\right] d u, \\
\Phi_{\alpha, \beta}(u, v)= & \frac{1}{u} \int_{0}^{u}\left(u v \frac{\partial G_{\alpha, \beta}(u, v)}{\partial u}+v G_{\alpha, \beta}(u, v)+\frac{d}{d u}\left[u \Phi_{\alpha}(u, 0)\right]\right) d u \\
& +\frac{1}{u} \int_{0}^{u} \frac{v}{u} S_{x}^{\alpha} S_{t}^{\beta}\left[\frac{\partial^{\alpha}}{\partial x^{\alpha}}\left(\frac{x^{\alpha}}{\alpha} \frac{\partial^{\alpha} \varphi}{\partial x^{\alpha}}\right)-\eta \frac{x^{\alpha}}{\alpha} \psi \frac{\partial^{\alpha} \varphi}{\partial x^{\alpha}}-\zeta \frac{x^{\alpha}}{\alpha} \frac{\partial^{\alpha}}{\partial x^{\alpha}}(\psi \varphi)\right] d u .
\end{aligned}
$$

Applying the inverse double Sumudu transform, to both sides of equation (62) yields the following:

$$
\begin{aligned}
\psi\left(\frac{x^{\alpha}}{\alpha}, \frac{t^{\beta}}{\beta}\right)= & S_{u}^{-1} S_{v}^{-1}\left[\frac{1}{u} \int_{0}^{u}\left(u v \frac{\partial F_{\alpha, \beta}(u, v)}{\partial u}+v F_{\alpha, \beta}(u, v)+\frac{d}{d u}\left[u \Psi_{\alpha}(u, 0)\right]\right) d u\right] \\
& +S_{u}^{-1} S_{v}^{-1}\left[\frac{1}{u} \int_{0}^{u} \frac{v}{u} S_{x}^{\alpha} S_{t}^{\beta}\left[\frac{\partial^{\alpha}}{\partial x^{\alpha}}\left(\frac{x^{\alpha}}{\alpha} \frac{\partial^{\alpha} \psi}{\partial x^{\alpha}}\right)-\eta \frac{x^{\alpha}}{\alpha} \psi \frac{\partial^{\alpha} \psi}{\partial x^{\alpha}}-\zeta \frac{x^{\alpha}}{\alpha} \frac{\partial^{\alpha}}{\partial x^{\alpha}}(\psi \varphi)\right] d u\right], \\
\varphi\left(\frac{x^{\alpha}}{\alpha}, \frac{t^{\beta}}{\beta}\right)= & S_{u}^{-1} S_{v}^{-1}\left[\frac{1}{u} \int_{0}^{u}\left(u v \frac{\partial G_{\alpha, \beta}(u, v)}{\partial u}+v G_{\alpha, \beta}(u, v)+\frac{d}{d u}\left[u \Phi_{\alpha}(u, 0)\right]\right) d u\right] \\
& +S_{u}^{-1} S_{v}^{-1}\left[\frac{1}{u} \int_{0}^{u} \frac{v}{u} S_{x}^{\alpha} S_{t}^{\beta}\left[\frac{\partial^{\alpha}}{\partial x^{\alpha}}\left(\frac{x^{\alpha}}{\alpha} \frac{\partial^{\alpha} \varphi}{\partial x^{\alpha}}\right)-\eta \frac{x^{\alpha}}{\alpha} \psi \frac{\partial^{\alpha} \varphi}{\partial x^{\alpha}}-\zeta \frac{x^{\alpha}}{\alpha} \frac{\partial^{\alpha}}{\partial x^{\alpha}}(\psi \varphi)\right] d u\right] .
\end{aligned}
$$

The conformable double Sumudu decomposition method proposes a series solution of the functions $\psi\left(x^{\alpha} / \alpha\right.$, $\left.t^{\beta} / \beta\right)$ and $\varphi\left(x^{\alpha} / \alpha, t^{\beta} / \beta\right)$ by the infinite series

$$
\begin{aligned}
\psi\left(\frac{x^{\alpha}}{\alpha}, \frac{t^{\beta}}{\beta}\right)=\sum_{n=0}^{\infty} & \psi_{n}\left(\frac{x^{\alpha}}{\alpha}, \frac{t^{\beta}}{\beta}\right), \varphi\left(\frac{x^{\alpha}}{\alpha}, \frac{t^{\beta}}{\beta}\right)=\sum_{n=0}^{\infty} \varphi_{n}\left(\frac{x^{\alpha}}{\alpha}, \frac{t^{\beta}}{\beta}\right), \\
\sum_{n=0}^{\infty} \psi_{n}\left(\frac{x^{\alpha}}{\alpha}, \frac{t^{\beta}}{\beta}\right)= & S_{u}^{-1} S_{v}^{-1}\left[\frac { 1 } { u } \int _ { 0 } ^ { u } \left(u v \frac{\partial F_{\alpha, \beta}(u, v)}{\partial u}+v F_{\alpha, \beta}(u, v)\right.\right. \\
& \left.\left.+\frac{d}{d u}\left[u \Psi_{\alpha}(u, 0)\right]\right) d u\right]+S_{u}^{-1} S_{v}^{-1} \\
& \cdot\left[\frac{1}{u} \int_{0}^{u}\left(\frac{v}{u} S_{x}^{\alpha} S_{t}^{\beta}\left[\frac{\partial^{\alpha}}{\partial x^{\alpha}}\left(\frac{x^{\alpha}}{\alpha} \frac{\partial^{\alpha}}{\partial x^{\alpha}}\left(\sum_{n=0}^{\infty} \varphi_{n}\right)\right)\right]\right) d u\right] \\
& -S_{u}^{-1} S_{v}^{-1}\left[\frac{1}{u} \int_{0}^{u}\left(\frac{v}{u} S_{x}^{\alpha} S_{t}^{\beta}\left[\eta \frac{x^{\alpha}}{\alpha} \sum_{n=0}^{\infty} A_{n}\right]\right) d u\right] \\
& -S_{u}^{-1} S_{v}^{-1}\left[\frac{1}{u} \int_{0}^{u}\left(\frac{v}{u} S_{x}^{\alpha} S_{t}^{\beta}\left[\zeta \frac{x^{\alpha}}{\alpha} \sum_{n=0}^{\infty} C_{n}\right]\right) d u\right],
\end{aligned}
$$

$$
\begin{aligned}
\sum_{n=0}^{\infty} \varphi_{n}\left(\frac{x^{\alpha}}{\alpha}, \frac{t^{\beta}}{\beta}\right)= & S_{u}^{-1} S_{v}^{-1}\left[\frac { 1 } { u } \int _ { 0 } ^ { u } \left(u v \frac{\partial G_{\alpha, \beta}(u, v)}{\partial u}+v G_{\alpha, \beta}(u, v)\right.\right. \\
& \left.\left.+\frac{d}{d u}\left[u \Phi_{\alpha}(u, 0)\right]\right) d u\right]+S_{u}^{-1} S_{v}^{-1} \\
& \cdot\left[\frac{1}{u} \int_{0}^{u}\left(\frac{v}{u} S_{x}^{\alpha} S_{t}^{\beta}\left[\frac{\partial^{\alpha}}{\partial x^{\alpha}}\left(\frac{x^{\alpha}}{\alpha} \frac{\partial^{\alpha}}{\partial x^{\alpha}}\left(\sum_{n=0}^{\infty} \varphi_{n}\right)\right)\right]\right) d u\right] \\
& -S_{u}^{-1} S_{v}^{-1}\left[\frac{1}{u} \int_{0}^{u}\left(\frac{v}{u} S_{x}^{\alpha} S_{t}^{\beta}\left[\eta \frac{x^{\alpha}}{\alpha} \sum_{n=0}^{\infty} B_{n}\right]\right) d u\right] \\
& -S_{u}^{-1} S_{v}^{-1}\left[\frac{1}{u} \int_{0}^{u}\left(\frac{v}{u} S_{x}^{\alpha} S_{t}^{\beta}\left[\mu \frac{x^{\alpha}}{\alpha} \sum_{n=0}^{\infty} C_{n}\right]\right) d u\right] .
\end{aligned}
$$

where $A_{n}, B_{n}$, and $C_{n}$ are given by equation (42). Consequently,

$\psi_{0}=S_{u}^{-1} S_{v}^{-1}\left[\frac{1}{u} \int_{0}^{u}\left(u v \frac{\partial F_{\alpha, \beta}(u, v)}{\partial u}+v F_{\alpha, \beta}(u, v)+\frac{d}{d u}\left[u \Psi_{\alpha}(u, 0)\right]\right) d u\right]$,
$\varphi_{0}=S_{u}^{-1} S_{v}^{-1}\left[\frac{1}{u} \int_{0}^{u}\left(u v \frac{\partial G_{\alpha, \beta}(u, v)}{\partial u}+v G_{\alpha, \beta}(u, v)+\frac{d}{d u}\left[u \Phi_{\alpha}(u, 0)\right]\right) d u\right]$,

$$
\begin{aligned}
\psi_{n+1}\left(\frac{x^{\alpha}}{\alpha}, \frac{t^{\beta}}{\beta}\right)= & S_{u}^{-1} S_{v}^{-1}\left[\frac{1}{u} \int_{0}^{u}\left(\frac{v}{u} S_{x}^{\alpha} S_{t}^{\beta}\left[\frac{\partial^{\alpha}}{\partial x^{\alpha}}\left(\frac{x^{\alpha}}{\alpha} \frac{\partial^{\alpha} \psi_{n}}{\partial x^{\alpha}}\right)\right]\right) d u\right] \\
& -S_{u}^{-1} S_{v}^{-1}\left[\frac{1}{u} \int_{0}^{u}\left(\frac{v}{u} S_{x}^{\alpha} S_{t}^{\beta}\left[\eta \frac{x^{\alpha}}{\alpha} A_{n}\right]\right) d u\right] \\
& -S_{u}^{-1} S_{v}^{-1}\left[\frac{1}{u} \int_{0}^{u}\left(\frac{v}{u} S_{x}^{\alpha} S_{t}^{\beta}\left[\zeta \frac{x^{\alpha}}{\alpha} C_{n}\right]\right) d u\right], \\
\varphi_{n+1}\left(\frac{x^{\alpha}}{\alpha}, \frac{t^{\beta}}{\beta}\right)= & S_{u}^{-1} S_{\mathrm{v}}^{-1}\left[\frac{1}{u} \int_{0}^{u}\left(\frac{v}{u} S_{x}^{\alpha} S_{t}^{\beta}\left[\frac{\partial^{\alpha}}{\partial x^{\alpha}}\left(\frac{x^{\alpha}}{\alpha} \frac{\partial^{\alpha} \varphi_{n}}{\partial x^{\alpha}}\right)\right]\right) d u\right] \\
& -S_{u}^{-1} S_{v}^{-1}\left[\frac{1}{u} \int_{0}^{u}\left(\frac{v}{u} S_{x}^{\alpha} S_{t}^{\beta}\left[\eta \frac{x^{\alpha}}{\alpha} B_{n}\right]\right) d u\right] \\
& -S_{u}^{-1} S_{v}^{-1}\left[\frac{1}{u} \int_{0}^{u}\left(\frac{v}{u} S_{x}^{\alpha} S_{t}^{\beta}\left[\mu \frac{x^{\alpha}}{\alpha} C_{n}\right]\right) d u\right] .
\end{aligned}
$$

Assume that double inverse Sumudu transform with respect $u$ and $v$ exists for each term in the right-hand side of equations (65), (66), and (67).

Example 12. Consider singular one-dimensional conformable fractional coupled burgers' equations

$$
\begin{aligned}
& \frac{\partial^{\beta} \psi}{\partial t^{\beta}}-\frac{\alpha}{x^{\alpha}} \frac{\partial^{\alpha}}{\partial x^{\alpha}}\left(\frac{x^{\alpha}}{\alpha} \frac{\partial^{\alpha} \psi}{\partial x^{\alpha}}\right)-2 \psi \frac{\partial^{\alpha} \psi}{\partial x^{\alpha}}+\frac{\partial^{\alpha}}{\partial x^{\alpha}}(\psi \varphi)=\left(\frac{x^{\alpha}}{\alpha}\right)^{2} e^{t^{\beta} \beta}-4 e^{t^{\beta} / \beta}, \\
& \frac{\partial^{\beta} \varphi}{\partial t^{\beta}}-\frac{\alpha}{x^{\alpha}} \frac{\partial^{\alpha}}{\partial x^{\alpha}}\left(\frac{x^{\alpha}}{\alpha} \frac{\partial^{\alpha} \varphi}{\partial x^{\alpha}}\right)-2 \varphi \frac{\partial^{\alpha} \varphi}{\partial x^{\alpha}}+\frac{\partial^{\alpha}}{\partial x^{\alpha}}(\psi \varphi)=\left(\frac{x^{\alpha}}{\alpha}\right)^{2} e^{t^{\beta} / \beta}-4 e^{\beta^{\beta} \beta},
\end{aligned}
$$


subject to

$$
\psi(x, 0)=\left(\frac{x^{\alpha}}{\alpha}\right)^{2}, \quad \varphi(x, 0)=\left(\frac{x^{\alpha}}{\alpha}\right)^{2}
$$

Using the above steps, we get

$$
\begin{aligned}
\sum_{n=0}^{\infty} \psi_{n}\left(\frac{x^{\alpha}}{\alpha}, \frac{t^{\beta}}{\beta}\right)= & \left(\frac{x^{\alpha}}{\alpha}\right)^{2} e^{t^{\beta} / \beta}-4 e^{t^{\beta} / \beta}+4-S_{u}^{-1} S_{v}^{-1} \\
& \cdot\left[\frac{1}{v} \int_{0}^{u}\left(S_{x}^{\alpha} S_{t}^{\beta}\left[\frac{\partial^{\alpha}}{\partial x^{\alpha}}\left(\frac{x^{\alpha}}{\alpha} \frac{\partial^{\alpha}}{\partial x^{\alpha}}\left(\sum_{n=0}^{\infty} \varphi_{n}\right)\right)\right]\right) d u\right] \\
& -S_{u}^{-1} S_{v}^{-1}\left[\frac{1}{v} \int_{0}^{u}\left(S_{x}^{\alpha} S_{t}^{\beta}\left[2 \frac{x^{\alpha}}{\alpha} \sum_{n=0}^{\infty} A_{n}\right]\right) d u\right]+S_{u}^{-1} S_{v}^{-1} \\
& \cdot\left[\frac{1}{v} \int_{0}^{u}\left(S_{x}^{\alpha} S_{t}^{\beta}\left[\frac{x^{\alpha}}{\alpha} \frac{\partial^{\alpha}}{\partial x^{\alpha}}\left(\sum_{n=0}^{\infty} C_{n}\right)\right]\right) d u\right] \\
\sum_{n=0}^{\infty} \varphi_{n}\left(\frac{x^{\alpha}}{\alpha}, \frac{t^{\beta}}{\beta}\right)= & \left.\left.\frac{x^{\alpha}}{\alpha}\right)^{2} e^{t^{\beta} / \beta}-4 e^{t^{\beta} / \beta}+4-S_{u}^{-1} S_{v}^{-1}\right] \\
& \cdot\left[\frac{1}{v} \int_{0}^{u}\left(S_{x}^{\alpha} S_{t}^{\beta}\left[\frac{\partial^{\alpha}}{\partial x^{\alpha}}\left(\frac{x^{\alpha}}{\alpha} \frac{\partial^{\alpha}}{\partial x^{\alpha}}\left(\sum_{n=0}^{\infty} \varphi_{n}\right)\right)\right]\right) d u\right] \\
& -S_{u}^{-1} S_{v}^{-1}\left[\frac{1}{v} \int_{0}^{u}\left(S_{x}^{\alpha} S_{t}^{\beta}\left[2 \frac{x^{\alpha}}{\alpha} \sum_{n=0}^{\infty} B_{n}\right]\right) d u\right]+S_{u}^{-1} S_{v}^{-1} \\
& \cdot\left[\frac{1}{v} \int_{0}^{u}\left(S_{x}^{\alpha} S_{t}^{\beta}\left[\frac{x^{\alpha}}{\alpha} \sum_{n=0}^{\infty} C_{n}\right]\right) d u\right],
\end{aligned}
$$

where the nonlinear terms $A_{n}, B_{n}$, and $C_{n}$ are denoted in equations (43), (44), and (45), respectively. In view of the recursive relations (65), (66), and (67), we obtained other components as follows:

$$
\begin{aligned}
& \psi_{0}=\left(\frac{x^{\alpha}}{\alpha}\right)^{2} e^{t^{\beta} / \beta}-4 e^{t^{\beta} / \beta}+4, \varphi_{0}=\left(\frac{x^{\alpha}}{\alpha}\right)^{2} e^{t^{\beta} / \beta}-4 e^{t^{\beta} / \beta}+4, \\
& \psi_{1}=-S_{u}^{-1} S_{v}^{-1}\left[\frac{1}{v} \int_{0}^{u} S_{x}^{\alpha} S_{t}^{\beta}\left[\frac{\partial^{\alpha}}{\partial x^{\alpha}}\left(\frac{x^{\alpha}}{\alpha} \frac{\partial^{\alpha} \psi_{0}}{\partial x^{\alpha}}\right)+2 \frac{x^{\alpha}}{\alpha} \psi_{0} \frac{\partial^{\alpha} \psi_{0}}{\partial x^{\alpha}}-\frac{x^{\alpha}}{\alpha} \frac{\partial^{\alpha}}{\partial x^{\alpha}}\left(\psi_{0} \varphi_{0}\right)\right] d u\right], \\
& \psi_{1}=-S_{u}^{-1} S_{v}^{-1}\left[\frac{1}{v} \int_{0}^{u} S_{x}^{\alpha} S_{t}^{\beta}\left[\left(4 \frac{x^{\alpha}}{\alpha} e^{\frac{t^{\beta}}{\beta}}\right)\right] d u\right]=4 e^{t^{\beta} / \beta}-4, \\
& \varphi_{1}=-S_{u}^{-1} S_{v}^{-1}\left[\frac{1}{v} \int_{0}^{u} S_{x}^{\alpha} S_{t}^{\beta}\left[\frac{\partial^{\alpha}}{\partial x^{\alpha}}\left(\frac{x^{\alpha}}{\alpha} \frac{\partial^{\alpha} \varphi_{0}}{\partial x^{\alpha}}\right)+2 \frac{x^{\alpha}}{\alpha} \varphi_{0} \frac{\partial^{\alpha} \varphi_{0}}{\partial x^{\alpha}}-\frac{x^{\alpha}}{\alpha} \frac{\partial^{\alpha}}{\partial x^{\alpha}}\left(\psi_{0} \varphi_{0}\right)\right] d u\right], \\
& \varphi_{1}=-S_{u}^{-1} S_{v}^{-1}\left[\frac{1}{v} \int_{0}^{u} S_{x}^{\alpha} S_{t}^{\beta}\left[\left(4 \frac{x^{\alpha}}{\alpha} e^{t^{\beta} / \beta}\right)\right] d u\right]=4 e^{t^{\beta} / \beta}-4, \\
& \psi_{2}=-S_{u}^{-1} S_{v}^{-1}\left[\frac{1}{v} \int_{0}^{u} S_{x}^{\alpha} S_{t}^{\beta}\left[\frac{\partial^{\alpha}}{\partial x^{\alpha}}\left(\frac{x^{\alpha}}{\alpha} \frac{\partial^{\alpha} \psi_{0}}{\partial x^{\alpha}}\right)\right] d u\right]-S_{u}^{-1} S_{v}^{-1} \\
& {\left[\frac{1}{v} \int_{0}^{u} S_{x}^{\alpha} S_{t}^{\beta}\left[2 \frac{x^{\alpha}}{\alpha}\left(\psi_{0} \frac{\partial^{\alpha} \psi_{1}}{\partial x^{\alpha}}+\psi_{1} \frac{\partial^{\alpha} \psi_{0}}{\partial x^{\alpha}}\right)\right] d u\right]+S_{u}^{-1} S_{v}^{-1} } \\
& \cdot\left[\frac{1}{v} \int_{0}^{u} S_{x}^{\alpha} S_{t}^{\beta}\left[\frac{x^{\alpha}}{\alpha} \frac{\partial^{\alpha}}{\partial x^{\alpha}}\left(\psi_{0} \varphi_{1}+\psi_{1} \varphi_{0}\right)\right] d u\right] \psi_{2}=0, \quad \varphi_{2}=0 .
\end{aligned}
$$

So the solution of singular one-dimensional conformable fractional coupled burgers' equations in the series form is given by

$\psi\left(\frac{x^{\alpha}}{\alpha}, \frac{t^{\beta}}{\beta}\right)=\psi_{0}+\psi_{1}+\psi_{2}+\cdots, \quad \varphi\left(\frac{x^{\alpha}}{\alpha}, \frac{t^{\beta}}{\beta}\right)=\varphi_{0}+\varphi_{1}+\varphi_{2}+\cdots$

Therefore, the exact solution is obtained as follows:

$$
\psi\left(\frac{x^{\alpha}}{\alpha}, \frac{t^{\beta}}{\beta}\right)=\left(\frac{x^{\alpha}}{\alpha}\right)^{2} e^{t^{\beta} / \beta} \text { and } \varphi\left(\frac{x^{\alpha}}{\alpha}, \frac{t^{\beta}}{\beta}\right)=\left(\frac{x^{\alpha}}{\alpha}\right)^{2} e^{t^{\beta} / \beta}
$$

By using $\alpha=1$ and $\beta=1$, the fractional solution becomes

$$
\psi(x, t)=x^{2} e^{t}, \quad \varphi(x, t)=x^{2} e^{t} .
$$

The exact solution of equation (68) for $\alpha=\beta=1$ is shown in Figure 3 (see also Figures 4(a)-4(c)) for $\alpha \neq \beta$.

\section{Conclusion}

In this paper, we introduce the conformable double Sumudu decomposition method and give the conformable double Sumudu transform of the fractional partial derivatives. Then we discuss the solution of regular and irregular onedimensional conformable fractional coupled burgers' equations by using a conformable double Sumudu decomposition method. Further properties and problems can be considered by using conformable double Sumudu decomposition. We sketched all the graphs by using Matlab. In the future, we are going to generalize conformable Laplace transform to solve more general nonlinear partial differential equations.

\section{Data Availability}

Data sharing not applicable to this article as no datasets were generated or analyzed during the current study.

\section{Conflicts of Interest}

The authors declare that there are no conflicts of interest regarding the publication of this paper.

\section{Authors' Contributions}

The authors read and approved the final manuscript.

\section{Acknowledgments}

The authors would like to extend their sincere appreciation to the Deanship of Scientific Research at King Saud University for funding this research group (No. RG-1440-030). 


\section{References}

[1] J. D. Cole, "On a quasilinear parabolic equation occuring in aerodynamics," Quarterly of Applied Mathematics, vol. 9, pp. 225-236, 1951.

[2] E. R. Benton and G. W. Platzman, "A table of solutions of the one-dimensional Burgers equation," Quarterly of Applied Mathematics, vol. 30, no. 2, pp. 195-212, 1972.

[3] A. Kurt, "New analytical and numerical results for fractional Bogoyavlensky-Konopelchenko equation arising in fluid dynamics," Applied Mathematics-A Journal of Chinese Universities, vol. 35, no. 1, pp. 101-112, 2020.

[4] A. Tozar, A. Kurt, and O. Tasbozan, "New wave solutions of an integrable dispersive wave equation with a fractional time derivative arising in ocean engineering models," Kuwait Journal of Science, vol. 47, no. 2, 2020.

[5] O. Tasbozan, "New analytical solutions for time fractional Benjamin-Ono equation arising internal waves in deep water," China Ocean Engineering, vol. 33, no. 5, pp. 593-600, 2019.

[6] A. Kurt, A. Tozar, and O. Tasbozan, "Applying the new extended direct algebraic method to solve the equation of obliquely interacting waves in shallow waters," Journal of Ocean University of China, vol. 19, no. 4, pp. 772-780, 2020.

[7] Q. D. Katatbeh and F. B. Belgacem, "Applications of the Sumudu transform to fractional differential equations," Nonlinear Studies, vol. 18, no. 1, pp. 99-112, 2011.

[8] D. Kumar, J. Singh, and A. Kiliçman, "An efficient approach for fractional Harry Dym equation by using Sumudu transform," Abstract and Applied Analysis, vol. 2013, Article ID 608943, 8 pages, 2013.

[9] Y. Çenesiz, D. Baleanu, A. Kurt, and O. Tasbozan, "New exact solutions of Burgers' type equations with conformable derivative," Wave Random Complex Media, vol. 27, pp. 103-116, 2016.

[10] J. Liu and G. Hou, "Numerical solutions of the space- and time-fractional coupled Burgers equations by generalized differential transform method," Applied Mathematics and Computation, vol. 217, no. 16, pp. 7001-7008, 2011.

[11] T. Abdeljawad, "On conformable fractional calculus," Journal of Computational and Applied Mathematics, vol. 279, pp. 5766, 2015.

[12] U. Katugampola, "A new fractional derivative with classical properties," 2014, http://arxiv.org/abs/1410.6535v2.

[13] R. Khalil, M. Al horani, A. Yousef, and M. Sababheh, "A new definition of fractional derivative," Journal of Computational Applied Mathematics, vol. 264, pp. 65-70, 2014.

[14] F. S. Silva, D. M. Moreira, and M. A. Moret, "Conformable Laplace transform of fractional differential equations," Axioms, vol. 7, no. 3, p. 55, 2018.

[15] O. Özkan and A. Kurt, "On conformable double Laplace transform," Optical and Quantum Electronics, vol. 50, no. 2, p. 103, 2018.

[16] H. Eltayeb, I. Bachar, and A. Kılıçman, "On conformable double Laplace transform and one dimensional fractional coupled Burgers' equation," Symmetry, vol. 11, p. 417, 2019.

[17] M. Eslami, "Exact traveling wave solutions to the fractional coupled nonlinear Schrodinger equations," Applied Mathematics and Computation, vol. 285, pp. 141-148, 2016.

[18] T. Abdeljawad, M. A. L. Horani, and R. Khalil, "Conformable fractional semigroups of operators," Journal of Semigroup Theory and Applications, vol. 2015, p. 7, 2015.
[19] O. Ozkan and A. Kurt, "Conformable fractional double Laplace transform and its applications to fractional partial integro-differential equations," Journal of Fractional Calculus and Applications, vol. 11, no. 1, pp. 70-81, 2020.

[20] H. Thabet and S. Kendre, "Analytical solutions for conformable space-time fractional partial differential equations via fractional differential transform," Chaos, Solitons and Fractals, vol. 109, pp. 238-245, 2018.

[21] H. Eltayeb, S. Mesloub, and A. Kılıçman, "A note on a singular coupled Burgers equation and double Laplace transform method," Journal of Nonlinear Sciences and Applications, vol. 11, pp. 635-643, 2018. 